\title{
A general framework for analyzing tumor subclonality using SNP array and DNA sequencing data
}

\author{
Bo $\mathrm{Li}^{1}$ and Jun $\mathrm{Z} \mathrm{Li}^{2^{*}}$
}

\begin{abstract}
Intra-tumor heterogeneity reflects cancer genome evolution and provides key information for diagnosis and treatment. When bulk tumor tissues are profiled for somatic copy number alterations (sCNA) and point mutations, it may be difficult to estimate their cellular fractions when a mutation falls within a sCNA. We present the Clonal Heterogeneity Analysis Tool, which estimates cellular fractions for both sCNAs and mutations, and uses their distributions to inform macroscopic clonal architecture. In a set of approximately 700 breast tumors, more than half appear to contain multiple recognizable aneuploid tumor clones, and many show subtype-specific differences in clonality for known cancer genes.
\end{abstract}

\section{Background}

It has been recognized for nearly 40 years that cancer is a dynamic disease and its evolution follows a classical Darwinian process [1,2]. After the proposal of the twohit model of oncogenesis [3], and especially after the discovery of the linear progression from benign polyps to colorectal cancer via a series of mutational events $[4,5]$, it was briefly envisioned that cancer could be understood in most cases by simply finding the small number of events that act sequentially to drive step-wise clonal selection. However, initial efforts to sequence most coding genes in tumor DNA revealed remarkable heterogeneity between tumors in each cancer type examined [6-9]: typically, very few $(<10)$ genes are mutated in $>10 \%$ of tumors, but many ( 40 to 80 ) are mutated in $1 \%$ to $5 \%$ of tumors. Further, heterogeneity in cancer could manifest on other levels: not just among different patients, but also among tumors of different grades or organ sites in the same patient, as well as among different cells within a tumor $[10,11]$. Heterogeneity at any of these levels could confound diagnosis and treatment, and underlie the inherent evasiveness of this disease. Most genomic analyses to date, notably those led by the Cancer Genome Atlas (TCGA) Research Network [12-15] and the

\footnotetext{
* Correspondence: junzli@med.umich.edu

${ }^{2}$ Department of Human Genetics, University of Michigan, 5940A Buhl, Box 5618, Ann Arbor, MI 48109-5618, USA

Full list of author information is available at the end of the article
}

International Cancer Genome Consortium (ICGC) [16] have focused on inter-tumor heterogeneity. These studies analyze hundreds of tumors per cancer type, relying on bulk tissue samples, usually for one sample per patient. The data were primarily interpreted by regarding each tumor as a single population of cells with uniform character. Despite the inherent limitation of this assumption, as shown by the widely reported tumor-normal mixing [17-19], large-scale inter-tumor comparisons have led to important new insights into significantly mutated genes $[12,13]$, recurrently perturbed pathways [20], mutation signatures [16,21], tumor subtypes [22,23], molecular predictors of outcome, and commonalities or distinctions among different cancer types [24]. However, these studies are not designed to adequately investigate intra-tumor heterogeneity. Ultimately, cancer genome evolution takes place at the single-cell level, and it is the cellular complexity and its dynamics that give rise to both intra- and inter-tumor heterogeneity. Currently, cytogenetic methods are of low throughput and often cannot assure representative sampling. And the cost of single-cell sequencing [25-28] remains prohibitively expensive for all but the proof-of-concept studies. Under such constraints, many groups have surveyed intra-tumor heterogeneity by comparing multiple specimens from the same patient by longitudinal sampling or spatial sampling (mainly for solid tumors). Almost invariably, analyses of longitudinal 
samples have uncovered dramatic temporal changes of the cancer cell population that often correlate with disease progression, severity, and treatment resistance [29-32]. Similarly, multi-region comparisons have revealed extensive genomic variability across different geographic sectors of the tumor $[33,34]$, or between the primary and metastatic tumors [35]. These studies, while using samples collected with a higher spatial or temporal resolution than those in TCGA and ICGC, often still contain heterogeneous populations of cells [35-37].

Fortunately, while bulk tissue data describe the global average of multiple subpopulations of cells, it is sometimes possible to statistically infer the number and genomic profile of such subpopulations. For example, when a sample is sequenced deeply, the somatic mutation frequencies sometimes cluster around a small number of distinct frequency 'modes' [38,39], suggesting that somatic mutations of similar frequencies may reside in the same population of cells and these cells may have descended from the same founder cell. For this reason, these mutations are said to belong to the same 'clone' or 'subclones', the latter referring to a clonal population of a relatively small cellular fraction. This inference task, essentially a deconvolution problem (or Blind Source Separation Problem), presents many analytical challenges, since both the number of subclones and the genomic profile of each need to be estimated simultaneously, and somatic copy number alterations (sCNAs) and somatic singlenucleotide variants (SNVs) often reside in the same region yet have unknown phase or genealogical order. Currently available methods often need to invoke simplifying assumptions and often focus on a subset of the issues. For example, ABSOLUTE [40] uses sCNA data to estimate the global mixing ratio of aneuploid and euploid cells, but only under a tumor-normal, two-population assumption, which involves a single tumor population of full clonality. When a sCNA or SNV is subclonal, ABSOLUTE makes the qualitative designation of 'subclonal' without quantitatively estimating the clonality. Other methods also invoke other types of compromises, and we will defer the description of these limitations to the Discussion.

In this work, we developed Clonal Heterogeneity Analysis Tool (CHAT) as a general framework for estimating the cellular frequencies of both SCNAs and SNVs. It is suitable for analyzing genomewide SNP genotyping and DNA sequencing data for tumor-normal pairs (Figure 1). CHAT begins by identifying regions of sCNA or by partitioning the genome into bins; and for each $\mathrm{SCNA}$ or bin, it estimates a local mixing ratio, called segmental aneuploid genome proportion (sAGP), between a euploid population and a single aneuploid population carrying the local CNA. The assumption of local two-way mixing does not imply there are only two cell populations globally. It is akin to the infinite-site model in population

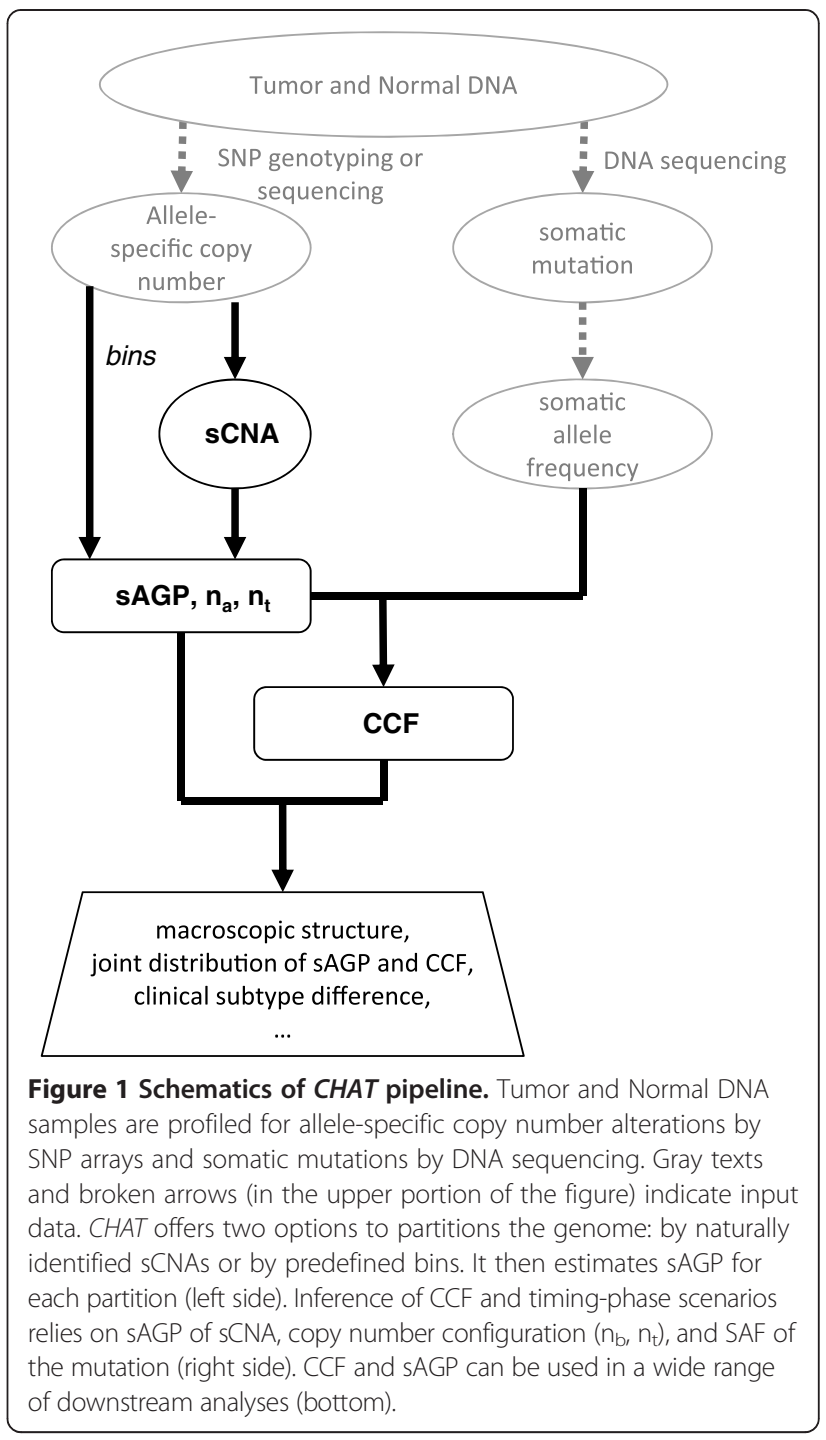

genetics, stating that each locus experienced only one copy number alteration, without a second over-riding alteration or the reversal to the original germline state (that is, back mutation). After calculating sAGP for every sCNA in the tumor, CHAT estimates the cellular prevalence of SNVs (also called cancer cell fraction, or CCF, as in [32]) by adjusting the observed somatic allele frequency (SAF) from sequencing data according to the background copy number status, while also considering the sCNA clonality (sAGP), the relative order of occurrence between the SNV and its associated sCNA, and their cis- or trans-relationship. Through simulation we show that CHAT performs well in quantitatively recovering sAGP, CCF, and the underlying evolutionary scenario. We also show that it estimates CCF more accurately than EXPANDS and PyClone in most scenarios and CNA states. We have applied CHAT to calculate sAGP for sCNAs, and CCF for SNVs, across 732 human breast 
tumor samples previously analyzed for inter-tumor diversity by TCGA [14] (Materials and methods, Data access and sCNA identification), and we will present two vignettes of the results. Lastly, we discuss the model identifiability issue and compare the theoretical features of CHAT with that of several similar methods.

\section{Results}

\section{Estimation of sAGP for sCNAs}

The simplest form of intra-tumor heterogeneity is normal cell 'contamination', that is, mixture of aneuploid cells in the tumor with euploid cells in the surrounding normal tissue, the latter carrying the full and balanced set of chromosomes found in germline DNA. In our previous work [18], we developed a method to calculate the overall fraction of the tumor cells, termed Aneuploid Genomic Proportion (AGP), assuming the global mixing of a tumor and a normal population. In brief, allelic intensity data from SNP genotyping arrays (or DNA sequencing) provide copy number information of the two parental chromosomes: $\mathrm{n}_{\mathrm{a}}$ and $\mathrm{n}_{\mathrm{b}}$. Since $\mathrm{n}_{\mathrm{a}}$ and $\mathrm{n}_{\mathrm{b}}$ are both integers, the logarithm of total intensity ratio, $L R R \sim \log \left(n_{a}+n_{b}\right)$, and the observed $B$ allele frequency, $B A F=n_{b} /\left(n_{a}+n_{b}\right)$, adopt a finite number of discrete BAF- LRR combinations for different CNAs, and reside in 'canonical positions' in the BAF-LRR plot. When aneuploid cells are mixed with euploid cells, logR-BAF positions of tumor sCNAs 'contract' towards the euploid position; and different mixing ratios result in different degrees of contraction. Based on this feature we can quantitatively estimate a genome-wide tumor mixing ratio [18]. Our algorithm relies on the same type of information, and shares the same goal, as several other methods (for example, ASCAT and ABSOLUTE) $[17,40]$. All of these methods assume that there is a single tumor population and use the combined information from all CNAs.

However, intra-tumor heterogeneity may also manifest as the co-existence of multiple tumor cell populations, each with its own copy number profile [41]. One example is shown in Figure 2A, where the sCNA segments marked in red show stronger contractions to the diploid track, for both LRR and BAF, than those marked in black; whereas those marked in green show even stronger contractions (Figure 2A and B). As mentioned above, since all the sCNAs in black have similar cellular fraction values, we may infer the existence of a subclone, defined as a subpopulation of cells carrying the same set of events (the 'black' sCNAs) due to their descent from a common ancestor tumor cell. This is the most parsimonious explanation why different somatic events in the genome could reach the same frequency. Meanwhile, another set of events, such as those in red, show a different cellular fraction values, suggesting the existence of a second subclone. Note that a subclone may be nested in a parental clone, and carry the events that are 'older' and of higher frequency. These 'parental events' may be shared between two sibling clones, each carrying its own unique set of newer events. Thus the sibling clones are disjoint (that is, non-overlapping) population of cells even when they share some events by common descent. Since the sCNA segments with different mixing ratios are interspersed along the genome, this regional variation of clonality motivates us to extend the earlier concept of genome-wide AGP to a new, segment-specific measure: sAGP.

A previous method [41] has attempted to simultaneously estimate the number of subpopulations, the copy number profile for each, and their mixing ratios. This deconvolution problem can be solved, in principle, via a general convex optimization algorithm, but in practice it is limited by computation burden, which increases quickly for more than several dozen events or more than three to four populations. Our method takes an alternative approach: CHAT estimates the mixing ratio for each sCNA (or bin) separately, postponing the question as to which events might belong to the same subclone by virtue of clustering around a similar sAGP, and how many subclones there might be. Thus, CHAT decouples the inference of local sAGPs from the subsequent clustering of sAGP, and is vastly more efficient: its computation time scales linearly with the number of sCNA events and there is nearly no time penalty when needing to consider an increasing number of subpopulations.

The estimation of sAGP follows a similar approach as estimating the global AGP [18], relying on the degree of contraction of each sCNA (Figure 2B, Materials and methods, sAGP inference). The method has the implicit assumption that at each sCNA the mixing involves only two populations, one of which is euploid. This assumption is largely satisfied when the somatic genome has experienced relatively sparse copy number changes, without global doubling or multiple rounds of complex local aberration. In effect, it assumes that, even though different sCNAs in the genome may belong to multiple populations of aneuploid cells, at each sCNA region there is only one aneuploid state that is mixed with the euploid state. As such, sAGP is a local quantity inferred for each SCNA, and is naturally assigned zero in regions with no sCNA. The input can be either SNP array data or sequencing data as long as there is a sufficient density of sites with allele-specific copy number data. At this step there is no need to determine if a sCNA is clonal or subclonal. Also of note is that in ABSOLUTE [40], subclones CNA events were described by a global purity value and a real-valued copy fraction (not an integer copy number). In contrast, CHAT explicitly models the mixing of a euploid population and an aneuploid population, involving a realvalued local mixing ratio and integer copy numbers. 

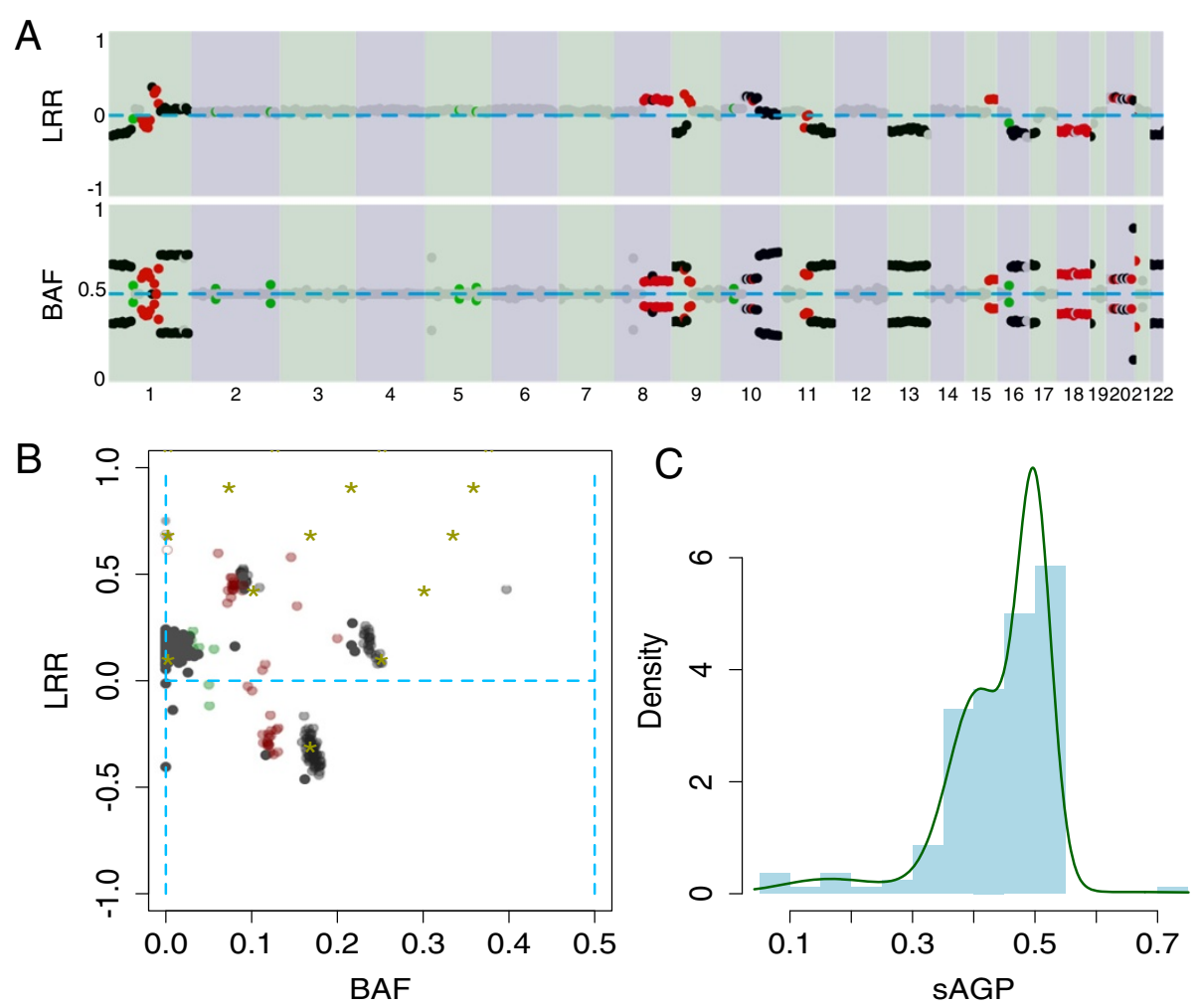

Figure 2 Example of intra-tumor heterogeneity (breast tumor sample TCGA-A1-A0SD). (A) BAF and LRR tracks for binned segments, showing different levels of contraction along the genome. Segments shown in the same color (black, red, green) have similar cellular fractions, and each may represent events in the same clonal population. (B) BAF-LRR plot for the same sample, showing different levels of contraction for segments of different colors. (C) MCMC fitting of SAGP distribution supports three modes, with peaks around SAGP $=0.5,0.4$, and 0.2 . The distribution of SAGP is indicated by the light blue histogram, while the fitted three-Gaussian density is shown in dark green.

\section{Macroscopic clonal structure}

Before describing the next step in CHAT - using sAGP and the observed SAF values to estimate CCF - we introduce an important downstream inference based on the genomewide distribution of sAGP values. When there are a sufficient number of sCNAs or bins covered by sCNA, CHAT produces a sufficient number of sAGP values; and their distribution could inform the clonal structure of the tumor. First, for some tumors the sAGP histogram may contain a single peak, potentially accompanied by a flat (nearly uniform) background distribution. This pattern can arise in a tumor containing a single clone that cover a large fraction of the sCNA-bearing portion of the genome, potentially with many other clones that cover much smaller portions of the genome and they are undiscernible in the sAGP spectrum. Second, for other tumors the histogram may follow a multi-modal distribution, representing a number of distinct clusters of somatic events, each with a different sAGP, with each cluster covering a comparable portion of the genome as to be recognizable in the histogram (an example is shown in Figure 2C).

In all, there are three attributes of each sAGP histogram. (1) The number of the identifiable modes corresponds to the number of identifiable cell populations. (2) The position of each mode denotes the cellular frequency of the sCNAs in each cluster, and reflects the clonality of the cell populations carrying these sCNAs. The right-most peak represents the sCNAs with the highest sAGP values; and they suggest the existence of a population of cells with the highest cellular fraction in the tumor. This population is typically called the dominant clone. The peaks to the left represent sCNAs with lower sAGP values and they are carried by populations of cells with lower cellular fractions. These populations are often called subclone 1, subclone 2 , and so on, but they may be nested within the dominant clone, and also carry the sCNAs in the rightmost peak. (3) The areas under the peaks reflect the number of the sCNAs, or the regularly spaced bins, that belong to each peak. Note that the right-most peak may not have the largest area, thus the dominant clone may not carry sCNAs that cover the widest portion of the genome.

There are at least two ways to define the spatial unit in the sAGP analysis, and CHAT provides both options (Materials and methods, Data access and sCNA identification). The first is to calculate sAGP for regularly spaced bins, either for a fixed window width or for a fixed number 
of SNPs. The resulting sAGP values resemble the conventional genetic 'markers'; and each tumor has a guaranteed number and density of such markers to construct the sAGP histogram, which is interpreted analogously to the allele frequency spectrum in population genetics studies. However, the bins do not match the naturally occurring sCNAs, which are highly variable in lengths, from tens of $\mathrm{kb}$ to entire chromosome arms. The sCNAs shorter than the bin width would have their true sAGP values 'diluted' by flanking euploid segments in the same bin; whereas those longer than the bin width would generated a string of correlated sAGP values as the same SCNA is artificially divided into multiple adjacent bins, thus violating the assumption that sAGPs are independent. In the second option, CHAT will apply the identified sCNA as the naturally occurring spatial unit for sAGP calculation. While this has the advantage that all sAGPs are truly independent, there are two disadvantages. First, the longer (or shorter) sCNAs provide more (or less) precise estimates of sAGP, but this information of confidence was discarded, as it is also the case in [41]. Two, there will be large tumor-tumor variations in the number of sCNAs, and some tumors may not have enough sCNAs to construct an informative histogram for estimating clonal composition. In short, the per-bin sAGPs (option 1) are derived from segments of similar length and have similar confidence intervals - they are identically distributed but not independent random variables. Conversely, the per-sCNA sAGPs (option 2) are independent, but are not identically distributed due to varying lengths. Rigorously speaking, neither is suited for analyzing macroscopic clonal architecture but can be applied in exploratory analysis, especially when there is no other data type such as the SNVs (see below).

When the primary goal of using CHAT is to accurately estimate CCF, which relies on accurate sAGP values, the user is advised to calculate sAGP using sCNAs as the spatial unit rather than the bins. Alternatively, when the primary goal is to explore clonal composition of a tumor, and if there are too few sCNAs and if most of them are very large, it is beneficial to increase the number of informative features, just as the detection of population stratification requires many ancestry informative markers. Here the user may choose regularly spaced bins to increase the number of available sAGPs. In fact, when sCNAs are few and large, it is more advisable to collect sequencing data; and if the mutation rate is high and/or the entire genome is sequenced (as opposed to small targeted regions), the number of SNVs may exceed that of sCNAs, and it is better to rely on the CCF histogram to estimate clonal structure. CCF distributions have the important advantage of meeting the condition of independent and identically distributed variable. Ultimately, the best approach is to integrate the sAGP and CCF distributions in estimating clonal structure.
CHAT fits the uni-modal pattern with a maximal likelihood framework, and the multi-modal pattern using a Bayesian Monte-Carlo Markov Chain (MCMC) approach, with Dirichlet Process prior to estimate a hierarchical Gaussian mixture model [42]. The approach is similar to those introduced in $[32,38,39,43]$. Details are provided in Materials and methods, Statistical modeling to infer macroscopic clonal structure. Model selection is based on the Bayesian Information Criterion (BIC) [44]. In Discussion we will further interpret the uni-modal and multi-modal patterns in terms of the likely evolutionary dynamics and the relationship to classic concepts such as punctuated equilibrium [45] and episodic evolution.

\section{Estimating cell fractions of somatic mutations Nature of the problem}

The next step of CHAT turns from estimating sAGP of sCNAs to estimating the frequency of cells carrying a specific mutation, that is, single nucleotide variant (SNV) or small insertion/deletion (indel). Here the method addresses the case where the tumor DNA has been sequenced, either for the whole genome or for a targeted subset, such as the exome. The input of the analysis is the observed number of reads in the sequence data containing the mutation as well as those containing the un-mutated allele. The relative fraction of mutation-bearing reads is termed somatic allele frequency (SAF). Following [32], we adopt CCF to denote the percentage of cells in the tumor sample carrying a specific somatic mutation. CCF is also termed cellular prevalence in [43]. The task is to use the observed SAF to estimate the unknown CCF.

If the mutation resides in a normal diploid region, it typically occurs on the background of one of the two parental chromosomes, contributing to about half the sequence reads in this region. In this simple case, as the fraction of cells carrying the mutation is CCF, the expected fraction of sequence reads carrying the mutation, SAF, is simply a binomial variable with an expected value of $\mathrm{CCF} / 2$. We therefore can estimate $\mathrm{CCF}$ by $\mathrm{SAF} \times 2$. However, if the mutation resides in a $\mathrm{sCNA}$, the relationship between CCF and SAF depends on the copy number configuration (for example, copy neutral loss of heterozygosity (CN-LOH), deletion, amplification, and so on) and its sAGP. Further, it also depends on the chromosomal background in which the mutation occurs. For example, in a region of heterozygous amplification where one of the chromosomes has been duplicated, if the mutation occurs on the duplicated chromosome, it will contribute twice the number of sequence reads than the case where it occurs on the un-duplicated chromosome. Lastly, if the mutation occurs after the duplication has happened and the duplication-bearing clone is undergoing expansion, only a subset of the duplication-bearing cells will carry the mutation, and the 
relative size of this subpopulation can be any value in 0 to $100 \%$ and will also affect the relationship between CCF and SAF. In the following we systematically consider these possible scenarios. We will make the parsimonious assumption that each mutation only occurred once in the evolutionary history of the tumor cell population, therefore we will ignore the possibility of recurrent mutation at the same position, or simultaneous emergence of the same mutation is different subpopulations of cells. We will treat SNVs and indels equally, and use the term 'mutation' to denote both.

\section{Order-phase scenarios between sCNA and SNV}

For a somatic mutation revealed by tumor DNA sequencing, with an observed SAF value, we consider the task of estimating CCF if this mutation resides in an SCNA, and the sCNA has been discovered by either SNP array genotyping data $[17,40]$ or by sequencing data $[32,38]$. We assume that the sCNA has been well characterized, such that we already know $\mathrm{n}_{\mathrm{a}}$ and $\mathrm{n}_{\mathrm{b}}$, the copy number of its major and minor alleles, respectively, that is, $n_{a} \geq n_{b}$, and $\mathrm{n}_{\mathrm{t}}=\mathrm{n}_{\mathrm{a}}+\mathrm{n}_{\mathrm{b}}$ is the total copy number. We also assume that its sAGP has been calculated using the method described above, and that SAF has been corrected for known sequencing errors and local biases [21,46]. Below we present the CCF estimation procedure for the case of heterozygous amplification $\left(\mathrm{n}_{\mathrm{a}}=2, \mathrm{n}_{\mathrm{b}}=1\right)$. The two other common sCNA types, heterozygous deletion $\left(\mathrm{n}_{\mathrm{a}}=1, \mathrm{n}_{\mathrm{b}}=0\right)$ and $\mathrm{CN}-\mathrm{LOH}\left(\mathrm{n}_{\mathrm{a}}=2, \mathrm{n}_{\mathrm{b}}=0\right)$, are described in Materials and methods, CCF estimation and scenario identifiability for $\mathrm{CN}-\mathrm{LOH}$ and deletion.

When a mutation resides in a sCNA region, there are three main scenarios that describe the possible mutationsCNA combinations in terms of their relative temporal order and the chromosomal background of the mutation (Figure 3):

A. The mutation and sCNA emerged sequentially, with the mutation occurring first, and the sCNA occurring in a subset of mutation-bearing cells (Figure 3A). This led to the co-existence of three subpopulations: the original euploid mutation-free cells, with the population fraction of $\mathrm{r}_{0}$; cells carrying the mutation only, with a fraction of $\mathrm{r}_{1}$; and cells carrying both the mutation and the $\mathrm{sCNA}\left(\mathrm{r}_{2}\right)$. The last subpopulation has two alternative outcomes: $\mathrm{A}_{1}$ : the duplication occurred on the mutation-bearing chromosome, and $\mathrm{A}_{2}$ : the duplication occurred on the mutation-free chromosome. Intuitively, $A_{1}$ will have higher SAF than $A_{2}$ with the same $\left(r_{0}, r_{1}, r_{2}\right)$ fractions.

B. Like $A$, the mutation and $\mathrm{sCNA}$ emerged sequentially; but unlike A, the sCNA occurred first, with the mutation occurring in a subset of sCNA-bearing cells (Figure 3B). Again we have three

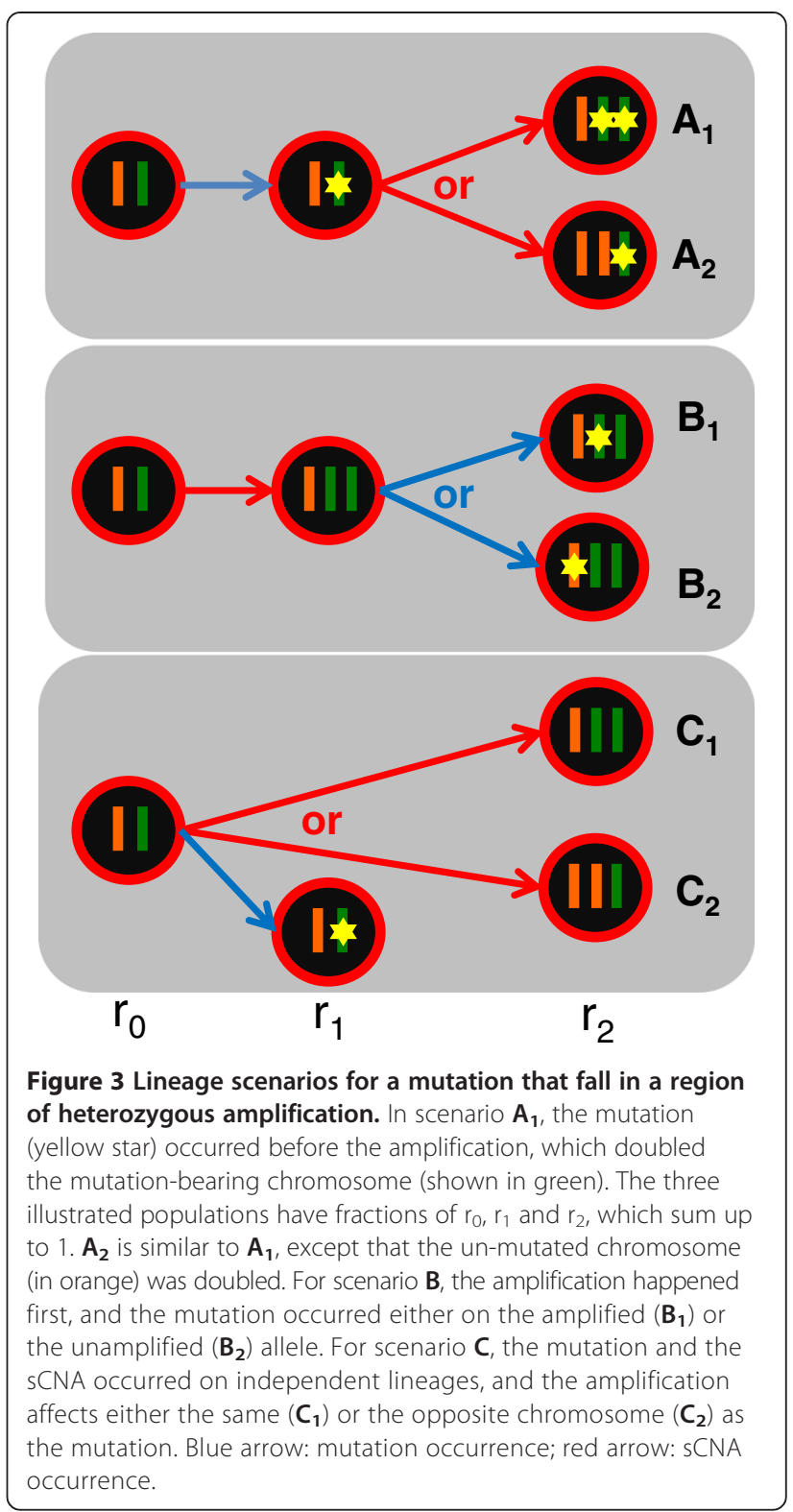

subpopulations: the original cells $\left(\mathrm{r}_{0}\right)$, cells carrying only the sCNA $\left(r_{1}\right)$ and those carrying both $\left(r_{2}\right)$. The last subpopulation has two alternatives: mutation occurring on one of the duplicated chromosome $\left(B_{1}\right)$ or the un-duplicated chromosome $\left(B_{2}\right)$.

$\mathrm{C}$. The mutation and sCNA emerged independently, that is, appearing in non-overlapping populations of cells (Figure 3C). This also led to three subpopulations: the original cells $\left(r_{0}\right)$, cells carrying only the mutation $\left(\mathrm{r}_{1}\right)$ and those carrying only the $\mathrm{sCNA}\left(\mathrm{r}_{2}\right)$. Note that we do not consider the fourth population that carries both the mutation and the sCNA. This outcome would require that the mutation occurred twice, once in the original cells and again in the sCNA-bearing cells. Or it 
requires the sCNA to occur twice. Under the Maximal Parsimonious assumption, recurrent appearance of the same mutation or the same sCNA is highly unlikely in the same tumor.

The three scenarios outlined above covered all the possible mutation-sCNA combinations for one-copy amplification without recurrence. In Additional file 1: Figure S1 and Materials and methods, CCF estimation and scenario identifiability for $\mathrm{CN}-\mathrm{LOH}$ and deletion, we show that heterozygous deletion and $\mathrm{CN}-\mathrm{LOH}$ involve similar scenarios $\mathbf{A}$, $\mathbf{B}$ and $\mathbf{C}$, and each leads to a similar set of three subpopulations as described by $r_{0}, r_{1}$, and $r_{2}$, with $r_{0}+r_{1}+r_{2}=1$.

\section{CCF as a function of SAGP, SAF and the underlying scenario}

When the $\left(\mathrm{n}_{\mathrm{a}}, \mathrm{n}_{\mathrm{b}}\right)$ configuration and evolutionary scenario is known, CCF can be estimated from: (1) the preestimated sAGP of the sCNA (denoted $p$ hereafter for simplicity) on which the mutation occurs; and (2) the observed allele frequency, SAF, of the somatic mutation (denoted $f$ hereafter). In the following we derive the CCF estimation procedure for heterozygous duplication $\left(\mathrm{n}_{\mathrm{a}}=2\right.$, $\left.\mathrm{n}_{\mathrm{b}}=1\right)$, and leave CN-LOH $\left(\mathrm{n}_{\mathrm{a}}=2, \mathrm{n}_{\mathrm{b}}=0\right)$, and deletion $\left(\mathrm{n}_{\mathrm{a}}=1, \mathrm{n}_{\mathrm{b}}=0\right)$ to Materials and methods, CCF estimation and scenario identifiability for $\mathrm{CN}-\mathrm{LOH}$ and deletion.

For amplification, in scenario $\mathbf{A}_{\mathbf{1}}, \mathrm{n}_{\mathrm{t}}=3$, the average total copy number $n_{t}=2 \times(1-p)+n_{t} \times p=2+p$. The sAGP $p=r_{2}$. The SAF $f=\left(r_{1}+2 r_{2}\right) /(2+p)$. This led to the expression $r_{1}=f \times(2+p)-2 r_{2}$. Since $C C F=r_{1}+r_{2}$, we have

$$
\begin{aligned}
C C F^{A_{1}}\left(f, n_{b}, n_{t}, p\right) & =f \times(2+p)-r_{2} \\
& =f \times(2+p)-p
\end{aligned}
$$

In $\mathbf{A}_{\mathbf{2}}$, the situation is similar to $\mathbf{A}_{\mathbf{1}}$ except that $f=$ $\left(r_{1}+r_{2}\right) /(2+p)$. This led to $r_{1}=f \times(2+p)-r_{2}$, and

$$
C C F^{A_{2}}\left(f, n_{b}, n_{t}, p\right)=f \times(2+p)
$$

In $\mathbf{B}_{\mathbf{1}}$ and $\mathbf{B}_{\mathbf{2}}$, the sAGP: $p=r_{1}+r_{2}$. The SAF: $f=r_{2} /(2+p)$. This led to $r_{2}=f \times(2+p)$. Since $C C F=r_{2}$, we have

$$
C C F^{B}\left(f, n_{b}, n_{t}, p\right)=f \times(2+p)
$$

In $\mathbf{C}_{\mathbf{1}}$ and $\mathbf{C}_{2}$, the sAGP: $p=r_{2}$. The SAF: $f=r_{1} /(2+p)$. This led to $r_{1}=f \times(2+p)$. Since $C C F=r_{1}$, we have

$$
C C F^{C}\left(f, n_{b}, n_{t}, p\right)=f \times(2+p)
$$

Note that equations (2), (3), and (4) are identical. Thus even if we do not know how to distinguish among scenarios $\mathbf{A}_{2}$, B, and $\mathbf{C}$, CCF still has the same dependency on sAGP and SAF, and can be estimated as long as we can recognize $\mathbf{A}_{\mathbf{1}}$ and $\mathbf{A}_{\mathbf{2}} / \mathbf{B} / \mathbf{C}$. Thus CCF identifiability is easier to achieve than scenario identifiability.

Similar expressions for $\mathrm{CN}-\mathrm{LOH}$ and deletion are presented in Materials and methods, CCF estimation and scenario identifiability for $\mathrm{CN}-\mathrm{LOH}$ and deletion. In the general copy number configuration of $\mathrm{n}_{\mathrm{a}}$ and $\mathrm{n}_{\mathrm{b}}$, for scenarios $A_{1}, A_{2}, B$, and $C$ we have

$$
\begin{aligned}
& C C F^{A_{1}}\left(f, n_{b}, n_{t}, p\right)=n_{t} \times f-p \times n_{a}+p \\
& C C F^{A_{2}}\left(f, n_{b}, n_{t}, p\right)=n_{t} \times f-p \times n_{b}+p \\
& C C F^{B / C}\left(f, n_{b}, n_{t}, p\right)=n_{t} \times f
\end{aligned}
$$

with $n_{t}=2 \times(1-p)+n_{t} \times p$, is the averaged copy number at the locus.

Thus, for a given pair of mutation and sCNA, with known SAF and sAGP values, once we know which scenario applies we can use Equations (1), (2), (3), (4), (5), (6), and (7) to estimate CCF. The variance of CCF estimates can be calculated as in Materials and methods, Variance of CCF. In the following we turn to the question of how to determine which scenario applies.

\section{Joint distribution of $(p, f)$ and scenario identifiability}

By definition, $f$ and $p$ are both bounded by $(0,1)$. In any tumor, however, the possible range of $f$ is constrained by $p$ as well as by the sCNA type and the individual scenarios. For example, in scenario $\mathbf{B}$ of amplification, the mutation occurs in a subset of sCNA-bearing cells, thus $f$ is always less than $p$ (in this case it is always less than $0.5 p$ ). As we show below, the attainable joint distributions of $(p, f)$ differs among different scenarios and, importantly, this offers the possibility to infer the most likely scenario for a given sCNA-mutation pair based on their observed $(p, f)$ values. Further, some 'zones' of the $(p, f)$ space overlap with multiple scenarios, thus if the observed $(p, f)$ fall into these zones, it is impossible to unambiguously identify the exact evolutionary scenarios. Even then, however, because different scenarios sometimes have the same expression of CCF as a function of $(p, f)$, CCF may still be uniquely estimated. In the following we derive the scenario-dependent $(p, f)$ joint distributions using heterozygous amplification as example.

In $\mathbf{A}_{1}$, for a given $p$, the observed $f$ of the mutation depends on the relative abundance of the $r_{0}$ and $r_{1}$ populations (Figure 3 ). When $r_{0}=0$, the mutation occurred so early that all the diploid cells carry the mutation and belong to the $\mathrm{r}_{1}$ subpopulation. $r_{1}=1-p$, and $f$ reaches its upper limit:

$$
f_{h}^{A_{1}}=\frac{1-p+2 \times p}{n_{t}}=\frac{1+p}{2+p}
$$

where $n_{t}=2 \times(1-p)+3 \times p$, is the averaged total copy number for the sCNA. On the opposite end of the situation is $r_{1}=0$, when the sCNA occurred immediately 
after the mutation such that none of the diploid cells carries the mutation. The lower limit of SAF is reached:

$$
f_{l}^{A_{1}}=\frac{2 p}{2+p}
$$

If we plot the possible $(p, f)$ combinations in an $p-f$ plot with $f$ on the vertical axis, under scenario $\mathbf{A}_{\mathbf{1}}$, the observed $\mathrm{f}$ is bounded by $(2 \mathrm{p} /(2+\mathrm{p}),(1+\mathrm{p}) /(2+\mathrm{p}))$, where $p \in(0,1)$, forming the zone marked $\mathbf{A}_{\mathbf{1}}$ in Figure 4A.

For $\mathbf{A}_{2}$, we similarly obtain:

$$
\begin{aligned}
& f_{h}^{A 2}=\frac{1-p+p}{n_{t}}=\frac{1}{2+p} \\
& f_{l}^{A 2}=\frac{p}{2+p}
\end{aligned}
$$

The observed $f$ for $\mathrm{A} 2$ is bounded by $(\mathrm{p} /(2+\mathrm{p}), 1 /(2+\mathrm{p}))$.

For $\mathbf{B}, f$ depends on the relative abundance of the $\mathrm{r}_{1}$ and $r_{2}$ populations, and the expressions are

$$
\begin{aligned}
f_{h}^{B} & =\frac{p}{n_{t}}=\frac{p}{2+p} \\
f_{l}^{B} & =0
\end{aligned}
$$

Thus $f$ is bounded by $[0, \mathrm{p} /(2+\mathrm{p})]$.
For $\mathbf{C}$, the upper limit of $f$ is reached when $\mathrm{r}_{0}=0$, $r_{1}=1-p$, and

$$
\begin{aligned}
f_{h}^{C} & =\frac{1-p}{n_{t}}=\frac{1-p}{2+p} \\
f_{l}^{C} & =0
\end{aligned}
$$

The $f$ is bounded by $[0,(1-\mathrm{p}) /(2+\mathrm{p})]$.

The results for $\mathrm{CN}-\mathrm{LOH}$ and deletion are described in Materials and methods, CCF estimation and scenario identifiability for $\mathrm{CN}-\mathrm{LOH}$ and deletion, and shown in Figure $4 \mathrm{~B}$ and $\mathrm{C}$.

To state the full estimation procedure: when $\left(f, n_{b}, n_{t}, p\right)$ are known for a mutation-sCNA pair, if the $(p, f)$ combination identifies a unique scenario according to Figure 4, CCF is calculated using Equations (5), (6), and (7). If the $(p, f)$ combination overlaps with multiple scenarios, CCF may still be calculated if the expressions are the same across the undistinguishable scenarios. Lastly, when the CCF expressions are different among the applicable scenarios, CCF cannot be uniquely determined, however its two or more possible values can still be obtained as valid alternatives. In implementation, as SAF is a random variable with confidence level depending on read depth, there is always uncertainty as to which scenario the observed $(p, f)$ belongs. We formally calculate the probability of each scenario as described in Materials and methods, Probabilistic scenario identification.
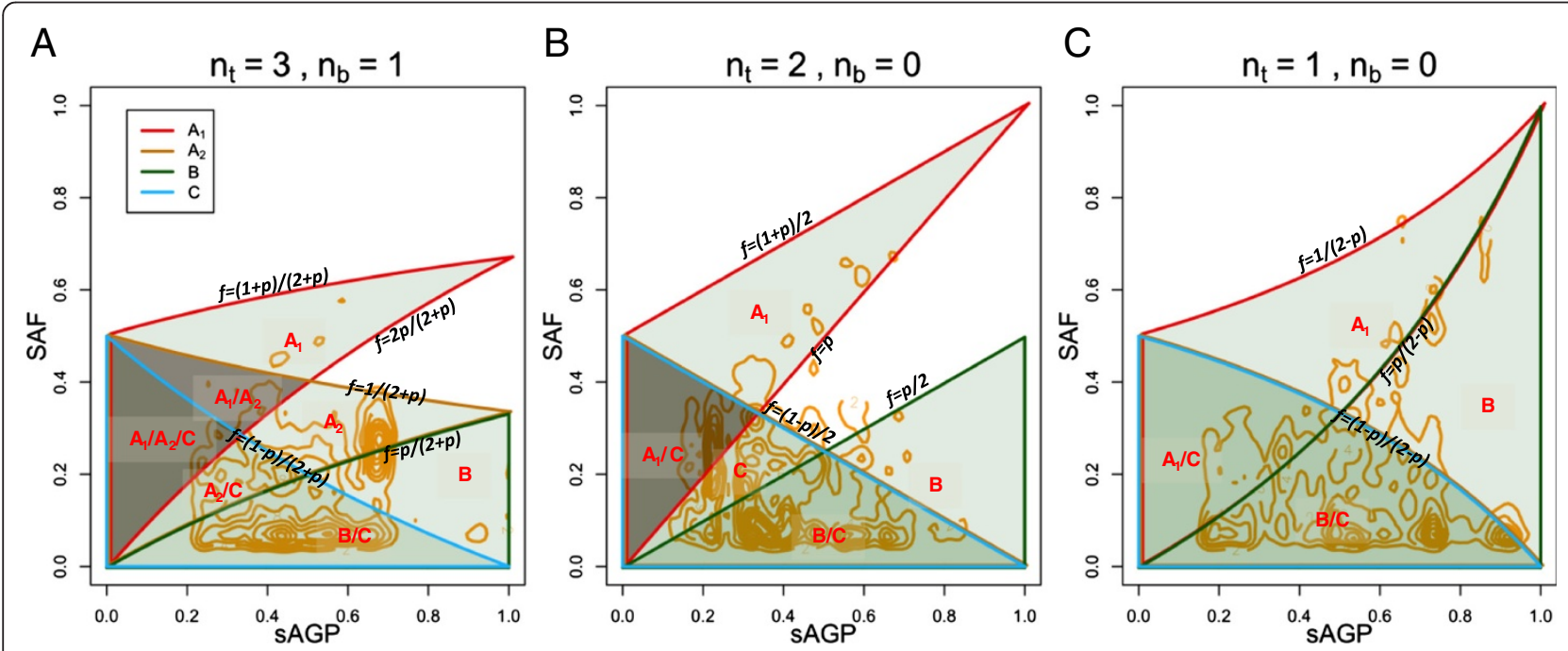

Figure 4 Identifiability zones in SAGP-CCF space, for amplification (A), CN-LOH (B) and deletion (C), with up to four scenarios described in the main text. Theoretically permissible areas of SAGP-CCF for different scenarios are marked by borders of different colors, and labeled with a single letter (such as ' $A_{1}$ ') for uniquely occupied zones, and by two or more letters (' $A_{1} / C^{\prime}$ ) for overlapping zones. Regions of light gray support a unique CCF expression, whereas the regions of dark gray cannot unambiguously estimate CCF. The density contours (in orange) depict the distribution of 3,382 mutations in amplification regions (A), 2,008 in CN-LOH, and 4,662 in deletions representing 201 breast tumor samples with least data loss in SAGP estimation. Variants with coverage lower than 20 or SAF smaller than 0.05 were discarded. Only a very small portion of the mutations fall outside the theoretically predicted zones. Among the rest, approximately $48 \%$ belong to a unique scenario, but approximately $93 \%$ have a unique CCF estimation. 


\section{Validation, implementation, and performance}

To assess the performance of CHAT we simulated sCNA and SNV data for a range of copy number configurations, sAGP values, evolutionary scenarios, and CCF values. Details of the simulation procedures were described in Materials and methods, In silico validation and computation performance. For sCNAs, we evaluated the performance by reporting: (1) percent of cases of mistaken estimation of sCNA configuration (error in either $\mathrm{n}_{\mathrm{b}}$ or $\mathrm{n}_{\mathrm{t}}$ ) (Figure 5A, top row) for dominant and minor clonal events; and (2) the median absolute deviation of the estimated sAGPs from the known sAGP values for the dominant and minor clones, for either the segments with correct $\left(n_{t}, n_{b}\right)$ identification (Figure $5 \mathrm{~A}$, middle row), or all segments (Figure 5A, bottom row). With all of these performance metrics, the errors are the largest when the clonal or subclonal sAGPs are small. The overall errors are small in most situations, suggesting that CHAT worked well in recovering the sAGP, $\mathrm{n}_{\mathrm{b}}$, and $\mathrm{n}_{\mathrm{t}}$ values. For $\mathrm{SNVs}$, we compared the estimated and the true CCF values in Figure 5B. Across all cases with different coverage and sCNA subclonal parameter settings, the Spearman's rank correlation coefficient between the known and the estimated CCF values was in the range of 0.946 to 0.97 , indicating that CHAT makes accuracy CCF inference. To compare performance among SNVs in different sub-categories, we separated those falling in euploid regions from those in sCNAs, and for the latter, separated those in the major and minor subclone events, and those in different copy number status. As shown in Figure $5 \mathrm{C}$, the error rates are similar across these sub-categories, not affected by dominant/minor clonal events or different sCNA types.

CHAT is written in R [47] and available as a CRAN package. It can use SNP array-based copy number data and sequencing-derived mutation data, or can use sequencing data as a single input source. It is computationally efficient, taking approximately 1 CPU-hour to analyze every five tumor-normal samples profiled with $850 \mathrm{~K}$ SNPs genotying and exome sequencing at $30 \times$ (Materials and methods, Computational requirements).

\section{Comparison with previous methods}

We compared CHAT with two other methods, EXPANDS and PyClone, that also estimate cellular frequencies for somatic mutations. We simulated 488 mutations that reside in sCNA regions and correspond to different linear scenarios and copy number states (details described in Materials and methods, Comparison with $E X$ PANDS and PyClone). Figure 6A, B, andC plots the CCF estimated by the three methods against the true CCF used to simulate the observed read counts. CHAT-based estimates have the highest correlation with the true CCF
(Pearson's $\mathrm{r}=0.96)$, followed by PyClone $(\mathrm{r}=0.94)$ and EXPANDS ( $\mathrm{r}=0.70)$. As the four lineage scenarios were distinguished by different symbol colors, it can be seen that PyClone underestimates CCF in scenarios $\mathrm{A}_{1}$ and $\mathrm{A}_{2}$ (Figure 6B), likely due to the assumption that mutation and sCNA always co-occur. PyClone performs similarly to CHAT in scenarios B and C. Like PyClone, EXPANDS also fails to consider the sCNA-free cells carrying the mutation, thus underestimates CCF in scenarios $\mathrm{A}_{1}$ and $\mathrm{A}_{2}$. EXPANDS overestimates CCF in $\mathrm{B}$ and $C$ (Figure $6 \mathrm{C}$ ) because it ignores $B$ and $C$ by applying $A_{1}$ or $A_{2}$ instead. To assess the impact of CNA status we further stratified the simulated mutations by individual combinations of lineage scenarios and CNA states, including deletion (genotype $\mathrm{A} / \mathrm{B}$ ), copy-neutral $\mathrm{LOH}(\mathrm{AA} / \mathrm{BB})$ and amplification $(\mathrm{AAB} / \mathrm{ABB})$ (Figure 6D). PyClone actually has a slight underestimation in scenario $\mathrm{B}$ for $\mathrm{CN}-\mathrm{LOH}$ and amplifications. The overestimation by EXPANDS in scenario $\mathrm{C}$ only occurs for deletions and amplifications. Overall, CHAT has the least bias and least variance in most combinations. Moreover, $C H A T$ is the most efficient. It took CHAT approximately $1 \mathrm{~s}$ to analyze the 488 somatic mutations. EXPANDS needed $732 \mathrm{~s}$, and PyClone took 4,320 s.

\section{Application to human breast cancer}

We applied CHAT to estimate sAGP for sCNAs identified using Affymetrix 6.0 single nucleotide polymorphism (SNP) array data for tumor and germline DNA samples from 732 breast cancer patients [14]. Of these, 445 also have whole-exome sequencing data available, and we estimated CCF for SNVs.

\section{sAGP distribution}

We detected sCNAs using circular binary segmentation [48] of LRR and BAF data [18], resulting in the identification of an average of 261 sCNAs per tumor (range: 1 to 3,537). The median size of all sCNAs is $1.7 \mathrm{Mb}$ (range: $2.5 \mathrm{~Kb}$ to $245 \mathrm{Mb}$ ). On average, each tumor carries $125 \mathrm{sCNAs}$ larger than $5 \mathrm{Mb}$, a size corresponding to approximately 1,500 SNP markers in the $850 \mathrm{~K} \mathrm{SNP}$ array. Given this sCNA size range, we re-calculated sAGP for genomic bins containing 500 heterozygous SNPs in the germline DNA, a bin size that is approximately $5 \mathrm{Mb}$, resulting in 502 bins per sample (range: 404 to 794) and constructed the sAGP histogram for every tumor. Eighty-seven tumors (12\%) had sCNAs for $<50$ bins, too few for analyzing the sAGP distribution patterns. For the remaining 645 tumors we fit the sCNAs distribution to either a uni-modal + uniform distribution or a multi-modal distribution using methods described in Materials and methods, Statistical modeling to infer macroscopic clonal structure. In the example in Figure $2 \mathrm{C}$, a three-mode distribution provides the 


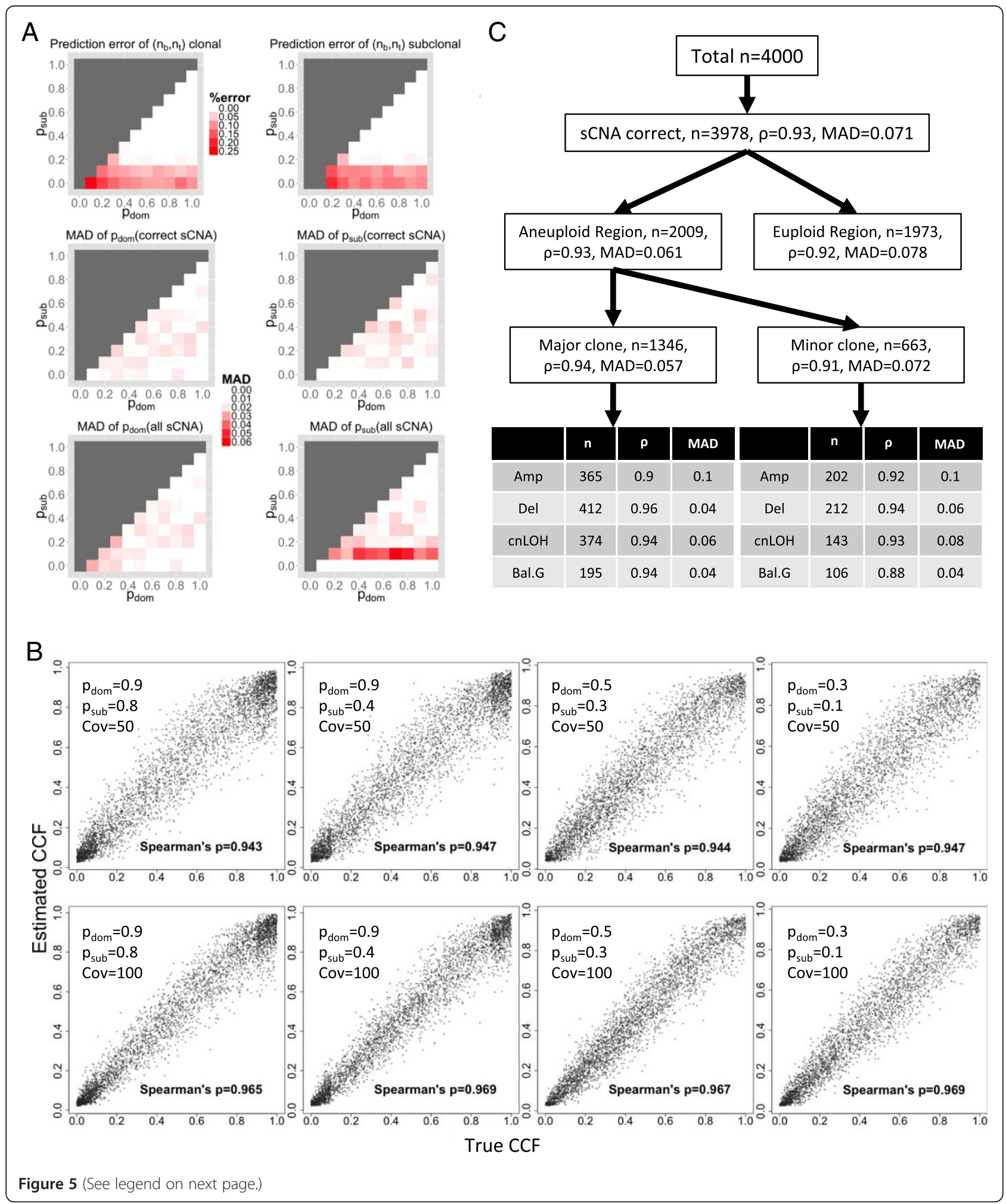


(See figure on previous page.)

Figure 5 In silico validation of CHAT performance. (A) Performance of SAGP inferences. Upper row: percent of error in estimated $n_{b}$ or $n_{t}$, for the dominant (left) and subclonal sCNAs (right), as described in Materials and methods, Performance of sAGP inference. Middle row: the median absolute difference (MAD) between estimated and simulated SAGP values for sCNAs with correctly identified ( $n_{b}$, $\left.n_{t}\right)$, or for all sCNAs (Bottom row). The $p_{\text {sub }}=0$ row of the lower-right and middle-right panels had zero error because when $p_{\text {sub }}=0$ there is only one clone in the tumor population and all subclonal sCNA segments have correctly estimated SAGP $=0$. (B) Performance of CCF inference. Shown are scatter plot of simulated and estimated CCF for four pdom - $p_{\text {sub }}$ Cases and two coverage values: Cov $=50$ (upper panels) and 100 (lower panels). (C) Comparison of CCF inference accuracy among different SNV categories: euploid vs. aneuploidy regions; and in the latter, between the dominant and the minor clones. Lastly, SNVs were divided by sCNA types. The tested case has the following parameter settings: $p_{\text {dom }}=0.9, p_{\text {sub }}=0.6$, coverage $=50$, number of SNV sampled $=4,000$, number of sCNA sampled $=200$. $\rho$, Spearman's correlation coefficient between the true and the estimated CCF values. MAD: median absolution difference between the true and the estimated CCF values.

best fit, with sAGP peaks around 0.5, 0.4, and 0.2. The highest peak corresponds to the black-colored sCNAs in Figure $2 \mathrm{~A}$ and $\mathrm{B}$, while the second and third peaks correspond to the red- and green-colored sCNAs, respectively. In total we observed 392 samples (61\%) with best fit by the multi-modal distribution, while 253 (39\%) follow the uni-model + uniform distribution. This shows that a majority of the breast tumors analyzed by TCGA contain more than one recognizable aneuploid population, suggesting that the co-existence of more than one subclone is very common.

\section{SAGP-CCF joint distribution for known cancer genes}

The 445 tumors with both SNP array and sequencing data have an average of 311 somatic mutations per tumor with CCF values (range: from 15 to 4,235, after counting the $8.8 \%$ loss due to sCNAs with un-estimable sAGP). While $48 \%$ of these mutations fall into a zone
A

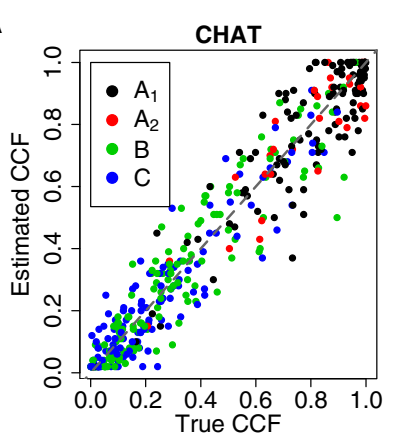

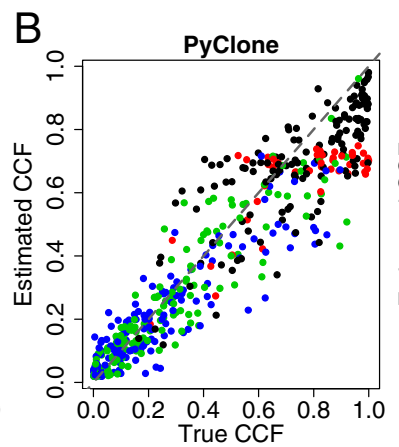
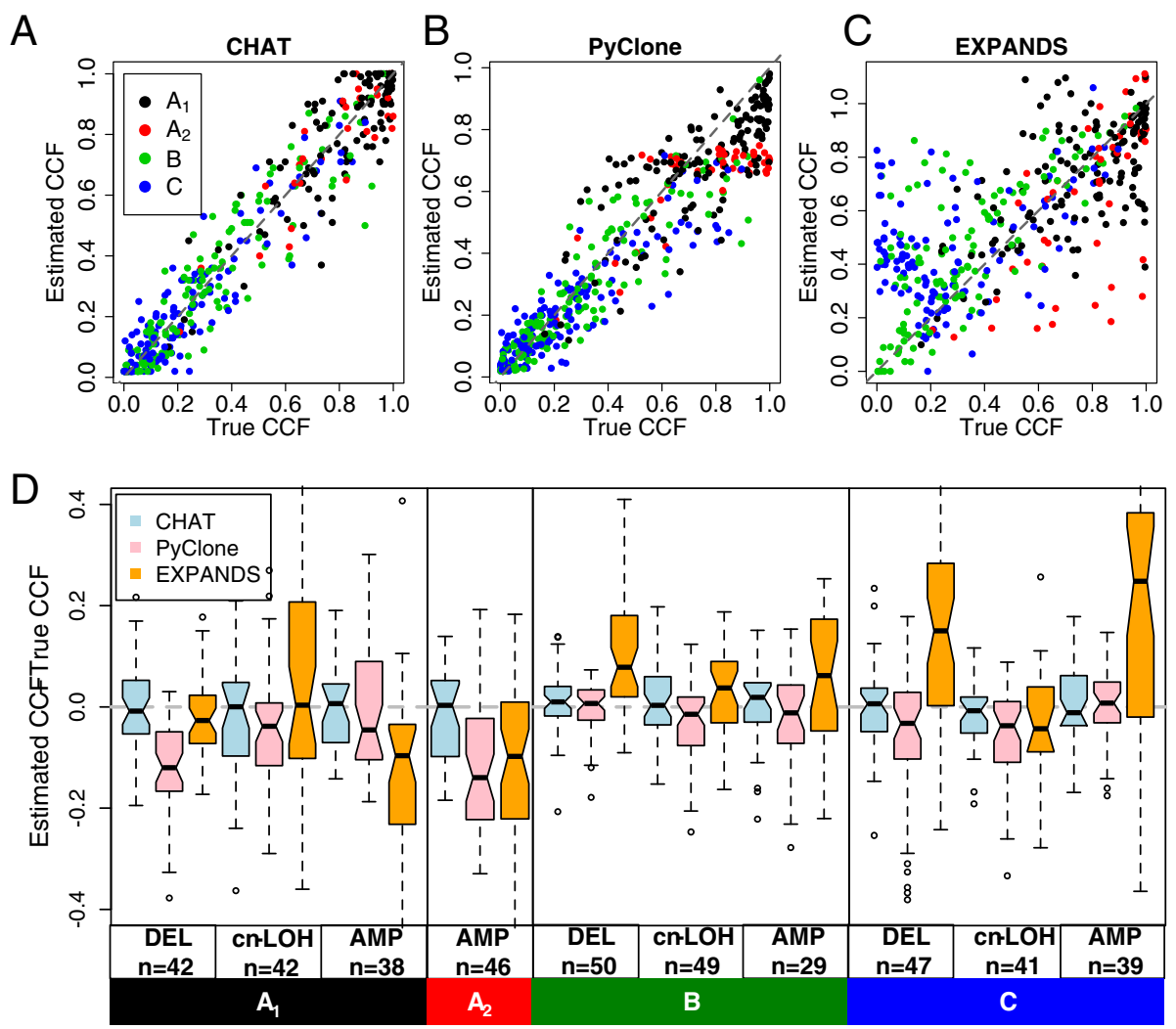

Figure 6 Performance comparisons of CHAT, EXPANDS, and PyClone. (A, B, C) Scatter plot of the CCF values estimated by the three methods against the known CCF values used in the simulation. The four lineage scenarios were distinguished by different symbol colors. (D) Boxplot showing the estimation errors for each of the three methods, stratified by both sCNA types and lineage scenarios, with the actual number of mutations simulated for each CNA type-scenario combination marked at the bottom of each panel. The sCNA types include heterozygous deletions (A/B), $\mathrm{cn}-\mathrm{LOH}(\mathrm{AA} / \mathrm{BB})$, and amplification (AAB/ABB). Mutations in balanced amplifications $(n=55)$ were not shown in order to reduce clutter. 
with overlapping scenarios, $93 \%$ of them have a unique mathematical expression and can produce a valid CCF estimate (Additional file 1: Figure S2). The remaining 7\% are assigned missing CCF values due to scenarios with conflicting CCF estimates.

The calculation of sAGP for most sCNAs and CCF for most SNVs makes it possible to examine the joint distribution of clonality for these two types of genome aberrations. A 'CCF vs. sAGP' plot can be created for all copy number and mutation events in a single tumor, or for events affecting a single gene of interest across many tumors. For a given gene, if a sample does not have any somatic mutation in the gene, we assign CCF $=0$. Likewise if the copy number of the gene is normal, we assign sAGP $=0$. Figure $7 \mathrm{~A}$ shows a heatmap depicting the density of CCF and sAGP joint distribution for all events in a hypothetical sample (or for a hypothetical gene across all samples). In this two-dimension space, the 'hot' peak near the origin $(0,0)$ is typical for most genes, affected by neither somatic mutation nor sCNA. The peak in the upper left (near the sAGP-axis) contains genes with highly clonal CNAs but carrying either no mutation or mutations of low clonality. A plausible interpretation is that for some of these genes, sCNA is a possible driver event. Similarly, the peak at the lower left (near the CCF-axis) contains genes with highly clonal somatic mutations and lowclonality sCNAs. Lastly, genes in the upper-right peak have both high sAGP and high CCF values, suggesting that both copy number changes and somatic mutations may have occurred at very early stages of tumor development, and their joint appearance may be necessary to act as a driver event.

Figure 7B allows close inspection of relative clonality between SCNA and mutations for four genes known to be related to breast cancer [14]: TP53, PIK3CA, and GATA3, which occurred in $>10 \%$ of analyzed breast tumors, and MAP3K1, which had mutations enriched in the luminal A subtype. For TP53, there are two noticeable high-density 'zones' in the heatmap: one along the sAGP-axis, the other at the upper right, indicating two groups of tumor samples: TP53 CNA-only and TP53 $\mathrm{CNA} /$ mutation, respectively. This pattern, when stratified by the four PAM50 subtypes [14,49] (Figure 7C), shows that the TP53 CNA/mutation group is enriched in the Basal and HER2 subtypes (accounting for 72 of 94 Basal and HER2 tumors), whereas the TP53 CNAonly group is enriched in the Luminal-A (94 of 105), and to a lesser degree, the Luminal-B subtypes (44 of 67). In comparison, the other three genes have not only the CNA-only and CNA/mutation groups, but also a third, mutation-only group near the CCF-axis. Figure 7D shows that for PIK3CA, the mutation-only group occurs almost exclusively in the Luminal-A and -B subtypes.
The CCF-sAGP plot can also be used to compare the clonality distribution between a pair of genes. In Figure 8, TP53 and PIK3CA are shown in red and blue symbols, respectively, with the lines linking the two genes for the same samples. There are three notable patterns of TP53-PIK3CA clonality. First, samples marked by the black lines have both sCNA and mutation in TP53 but no aberration in PIK3CA. Second, samples marked by the red and green lines tend to have sCNA for both TP53 and PIK3CA and at comparable sAGP, but only mutation in TP53 (red lines) or PIK3CA (green). Third, samples marked by the blue lines had high clonality for TP53 CNA, but not its mutation, and high clonality for PIK3CA mutation, but not its CNA, suggesting co-occurrence of aberrations of these two genes but involving different variant types. These patterns are subtype-specific: Pattern 1 is enriched in the Basal subtype $(\mathrm{OR}=4.6$ compared to the other three subtypes, $P=0.0001$ by Fisher's exact test, for red; OR $=1.2, P=0.67$, for green), so is Pattern $2(\mathrm{OR}=5.3, P=6.4 \mathrm{e}-8$,). Most remarkably, Pattern 3 is almost exclusively found in the Luminal A subtype $(\mathrm{OR}=56, P=4.4 \mathrm{e}-9)$.

\section{Discussion}

In this work, we developed a computational framework to estimate clonality for both sCNAs and SNVs. It is built on previous methods both by us [18] and by others $[32,38,41,43,50]$. While CHAT does not solve all the issues facing the cancer genome deconvolution problem, it attempts to overcome several important compromises or simplifying assumptions that underlie other methods. First, oncoSNP [51] and THetA [41] are designed to estimate sCNA clonality, but they do not address the clonality of somatic mutations. Second, Ding et al. [52] used a kernel density estimation method to characterize somatic mutations, but only focused on those in the euploid regions of genome, staying clear of the complicated relationship between SNV and sCNAs. Third, ABSOLUTE infers clonality for both sCNA and mutations but only designate subclonal status of the events, stopping short of quantitative estimation. This method was extended in Landau et al. [32] to estimate CCF for somatic mutations even if they are subclonal, but the algorithm only considers the case where sCNA occurred before SNV, equivalent to our scenario B (Figure 3 and Additional file 1: Figure S1), and further assumes that the copy number was altered by only one in the sCNA. In this regard, CHAT considers a wider array of possible scenarios. Fourth, $E X-$ $P A N D S$ [50] works with next-generation sequencing data and jointly estimates the absolute DNA copy number, clonality of somatic mutations, and that of sCNAs. However, this method only considers scenario A, and 


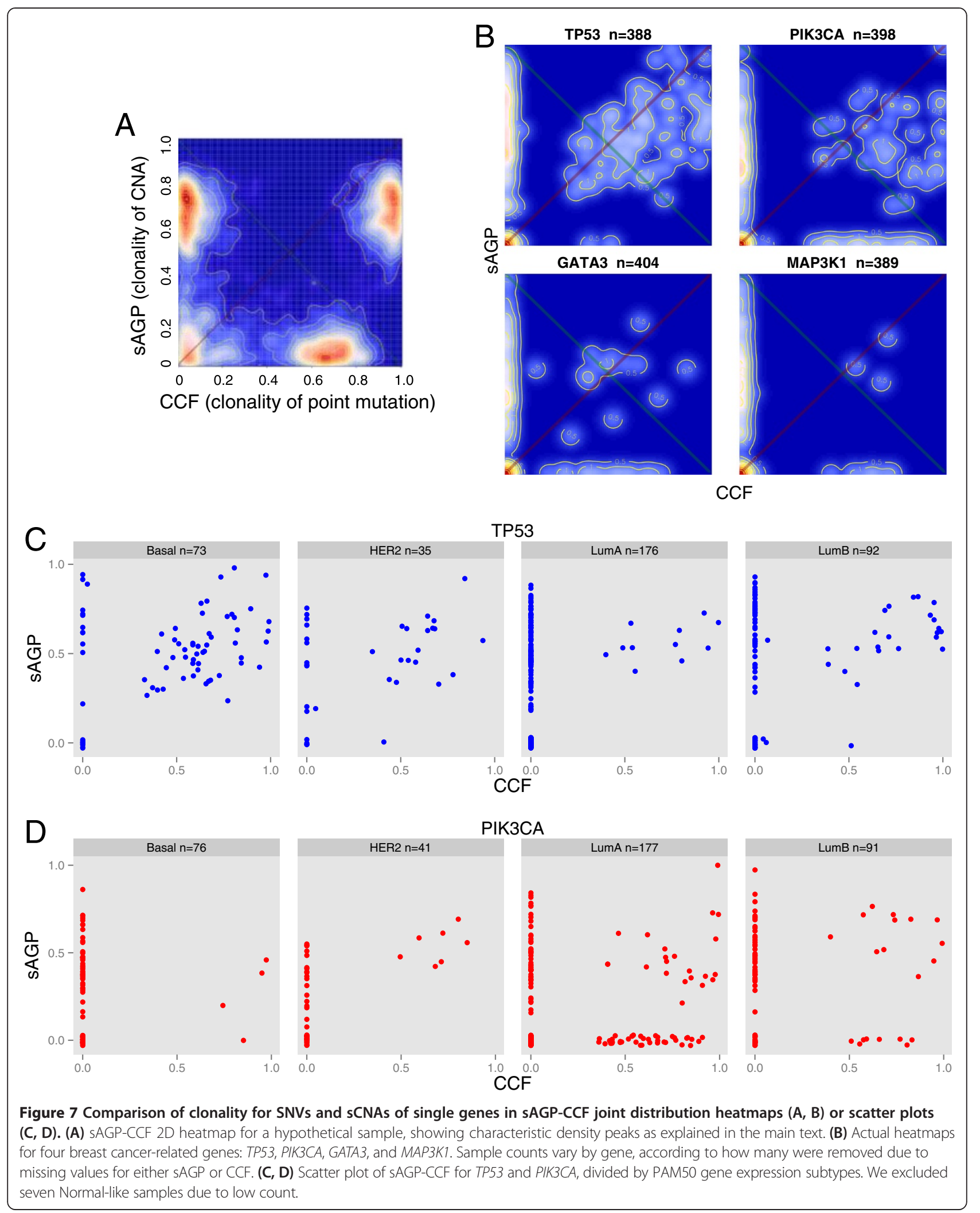




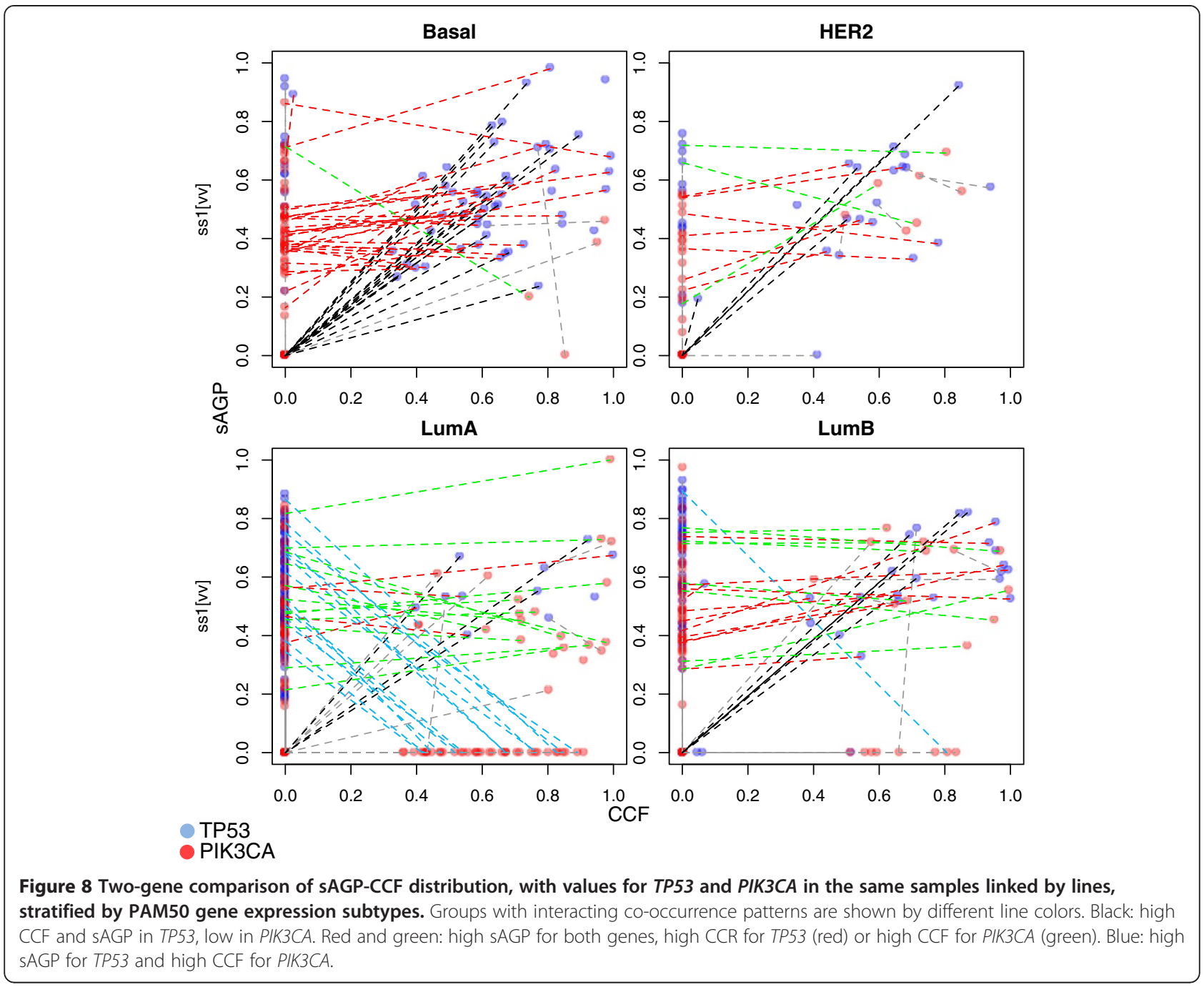

without the $r_{1}$ population. In effect, it assumes that the mutation and sCNA occur at the same instance and are in phase. These assumptions explained its underestimation of CCF in scenario A and overestimation in B and $\mathrm{C}$ (Figure 6). Fifth, PyClone [43] infers clonality of somatic mutations and performs phylogenetic analysis. It receives as input the integer copy number profiles estimated from other methods, but only considers scenarios $\mathrm{A}$ and $\mathrm{B}$, disregarding the possibility of a branching lineage (scenario C). Furthermore, for scenario A, it assumes co-occurrence of SNV and SCNA, thus ignoring the $\mathrm{r}_{1}$ population. In sum, the first key contribution of CHAT is in providing a general mathematical framework that enumerates the complete set of scenarios covering the possible order and phase of the sCNA and the mutation.

Like many of the previous methods, CHAT has its own limitations, primarily in being unable to resolve extremely complex events such as three-way mixing or above. It models two-population mixing at each genomic region (a gene, a sCNA, or a bin) and works best when the tumor has not experienced extensive and repeated copy number alterations. In the TCGA breast tumor dataset we found that $9.3 \%$ of sCNAs do not follow the regional two-way mixing model and preclude sAGP estimation. For the other, permissible sCNAs, CHAT can proceed, and is able to infer the co-existence of two or more subpopulations by analyzing the distribution of sAGP or CCF values. We wish to re-emphasize that while CHAT invokes two-way mixing for each individual genomic region, it is not limited to infer the presence of only two populations of cells. Globally, the number of peaks in the sAGP or CCF distribution has no restriction, and can be very high when the signal-to-noise ratio is improved, such as with ultra-deep sequencing data, for example, [39]. 
A second contribution of CHAT is in systematically assessing the input data combinations that leads to 'unidentifiable zones', in which the CCF, or 'scenarios' (that is, the evolutionary order and phase of the sCNA and SNV), cannot be resolved even with perfect data. Importantly, we found that in many situations, even if the evolution scenario is undetermined, CCF can still be estimated. The ability to objectively evaluate the power of inference in any given dataset is an important part of method development. Our treatment of this topic therefore sets useful constraints for future development of similar analysis tools. We found that, with the TCGA breast tumor data, about $9.3 \%$ of the identified sCNAs cannot be explained by local two-way mixing and were assigned missing sAGP values. In the second step, about $7 \%$ of the SNVs have unidentifiable CCF because they fall in either an inadmissible sAGPSAF zone or a region with conflicting CCF estimates. Thus 93\% of SNVs are suitable for CCF estimation, despite the fact that $48 \%$ of them involve ambiguous scenarios.

When applying CHAT to the breast tumor dataset, we found that approximately $61 \%$ of the breast tumors in the TCGA cohort contain more than one recognizable sAGP peaks, suggesting that even in a tumor cohort collected for studying inter-tumor diversity there is opportunity to detect intra-tumor mixing of multiple populations of aneuploid cells. And the results show that extensive intra-tumor heterogeneity does exist. This observation expands the earlier view that tumor-normal mixing contributes to intra-tumor heterogeneity, and confirms the results from single-cell and multi-region analyses in other solid tumors, for example, $[25,33]$. We wish to point out that the estimate of $61 \%$ was based on a specific analysis approach, and would vary with alternative parameter choices. For example, by using regularly spaced bins we found that 392 of 645 samples (61\%) were multi-modal, yet by using the naturally occurring sCNAs, only 635 samples had sufficient number of events, and 373 of them, or $59 \%$, were multimodal. More notably, of the 392 multi-modal samples called with bins, and the 373 multi-modal samples called with sCNAs, the overlap is 235 , or about $60 \%$ for either method. This level of concordance is related to the inherent shortage of observations for many samples: when the number of sCNAs or bins is in the 50 to 100 range, and if the primary peak is far larger than the secondary peak, the inference is less stable. These data-derived limitations can be overcome in the future when more samples are analyzed with whole-genome sequencing, which will likely yield a far greater number of genealogically informative markers.
A useful downstream analysis of the inferred clonality measures is to assess the distribution patterns of cellular frequencies of somatic aberrations, and to detect frequency clusters when they do exist for a given tumor. CHAT provides the option of characterizing the macroscopic clonal structure by a cluster-based approach (Figure 1). It is important to emphasize that these frequency clusters, despite their many valid interpretations, cannot be equated to individual subclones. A subclone may carry somatic events in multiple clusters, and may share some events with another subclone if they are descendants of the same parental clone. The full deconvolution of the observed aggregate pattern into those contributed by individual subclones requires further mathematical modeling and involves additional challenges. Several methods have recently appeared to address this 'Blind Source Separation Problem', or synonymously, 'Feature Allocation Problem' [53-56]. CHAT can be applied in tandem with these methods, that is, the sAGP and CCF output from CHAT can serve as the input data for further feature allocation to component subclones.

The co-existence of multiple clonal populations in bulk tissues could be explained by several population genomics models that are not mutually exclusive. First, in a multi-region parallel-evolution model, the tumor tissue might contain geographically segregated 'pure' populations, reflecting branched evolution of multiple clones of homogeneous tumor cells, each developing a different genomic profile that reflects its cell type of origin and adaptation to the local tissue habitat. This model can only be tested by spatially restricted sampling. Second, even in the absence of spatial segregation, the non-spatial, sequential expansion model could still lead to multiple nested populations. In some episodes, a burst of mutations or copy number variants might occur in one cell, which subsequently expands to a detectable clonal size driven by its unusually high selective advantage [57-59]. Alternatively, even in the absence of such disruptive genomic crisis, the slow, successive replacement of mildly advantageous clones could also result in a series of partial sweeps, leading to co-existence of multiple clones at any given time [60,61]. In other words, episodic acceleration of cancer genome evolution can take place either via mutation rate 'spikes' or simply through variabilities of selective advantage among driven events within a constant mutation regime. There are many routes that could lead from gradual evolution to punctuated equilibrium [62] in the history of each cell population; and this temporal heterogeneity is often further compounded in solid tumors by their spatial heterogeneity. 


\section{Conclusion}

We developed an automated pipeline that estimates cellular fractions for both sCNAs and mutations, and uses their distributions to inform macroscopic clonal architecture. It considers a wider range of evolutionary scenarios than existing methods concerning the timing and phase relationship between a sCNA and a mutation it contains. Our method also explicitly evaluates model- and parameter- identifiability. When applied to a previously analyzed set of $>700$ breast tumors we found more than half of the tumors appear to contain multiple recognizable aneuploid tumor clones, and many show subtype-specific differences in clonality between sCNA and mutation in known cancer genes. This method adds to the available toolkit for examining intra-tumor heterogeneity using bulk tumor genomic data.

\section{Materials and methods \\ Data access and SCNA identification}

From the Cancer Genome Atlas Data Portal [63] we downloaded: (1) the Level-2 copy number data derived from the Affymetrix Genome-Wide Human SNP Array 6.0 (the 'XX-byallele.copynumber.data.txt' files) for 732 breast tumor DNA and their paired normal tissue DNA; and (2) the VCF files for whole-exome sequencing data for a subset of 522 tumor-normal samples analyzed by TCGA [14]. The IDs of the 732 samples are in Additional file 2. Of these, 445 samples have both SNP array and DNA sequencing available. The SNP array data were downloaded on 12 December 2012, while the sequencing data were downloaded on 22 March 2013. Each VCF file contains variant information for both the tumor and the paired normal sample. The procedures for variant calling and identification of somatic variants can be found in the Online Supplementary Methods of [14]. Counts for somatic and reference alleles of both tumor and normal samples were extracted for use in this study.

In addition, we also downloaded the clinical annotation file, including the PAM50 designations of all the involved patients, on 17 December 2012.

sAGP estimation (see below) can be performed on two types of user-selected spatial units: (1) genomic bins, predefined for each sample, typically consisting of 500 heterozygous markers in the germline DNA; (2) naturally observed sCNA segments, which we detect using the Circular Binary Segmentation (CBS) method [48], as follows. We independently perform segmentation on the LRR and the folded BAF (absolute value of BAF minus 0.5) values, using default parameters in the $\mathrm{R}$ package DNAcopy [46], except that 'minimal markers required' was set to 5. With CBS results for both LRR and BAF, the two sets of change points are merged as follows: if a BAF change point falls within 5 markers of an LRR change point, either upstream or downstream, it is removed, that is, only the LRR breakpoint is kept, under the assumption that the two change points capture the same event, but the BAF change point is less accurately placed due to the greater sparsity of heterozygous markers.

After merging, the mean of LRR and folded BAF values are computed for each DNA segment (or the bin) in each sample, and used as input data for AGP and sAGP inference in the next step. For binned files, the bin length is on average $5.1 \mathrm{Mb}$, and each sample has an average of 502 bins.

\section{sAGP inference}

As discussed in the main text, we jointly use BAF and LRR values to estimate sAGP for each sCNA, under the assumption of regional two-way mixing. The algorithm has three steps:

\section{i. Data pre-processing}

We assume the allele-specific copy number data are already in bi-allelic format, with the following fields in the input file: SNP ID, chromosome, position, A allele count, B allele count. To note, the allele counts may not be integer numbers, but could be real-numbered values from the original CEL file. SNP markers are first grouped into either bins or merged sCNAs as described above. For each bin/sCNA, the median LRR and median folded BAF are calculated, and a segmentation file containing the above information for each segment is generated for each sample.

In the initial normalization of SNP array data the absolute LRR values depend on the genome-wide average ploidy, which is affected by the relative abundance of different copy number states in the genome. For example, in a tumor with a high fraction of cells undergone genome-wide doubling, the DNA segment located near the origin of the BAF-LRR plot are AABB, instead of the normal diploid configuration $\mathrm{AB}$, and the global ploidy can be well above 2 . The first step of sAGP estimation is therefore to ascertain the genotype of the sCNAs near the origin, following the procedures described in [18]. This allows unambiguous assignment (when possible) of copy number states for other sCNAs in the genome and the calculation of average ploidy. The deviation of BAF and LRR values of the baseline sCNAs from $\left(\mathrm{x}_{0}, \mathrm{y}_{0}\right)$ is also used to quantify $s d_{B A F}^{2}$ and $s d_{L R R}^{2}$ for use in downstream analysis.

\section{ii. Estimate sAGP and absolute copy numbers}


The method we used to estimate sAGP is extended from our AGP inference algorithm. For a sCNA with copy number configuration $\left(\mathrm{n}_{\mathrm{b}}, \mathrm{n}_{\mathrm{t}}\right)$, where $\mathrm{n}_{\mathrm{b}}$ is number of minor allele, and $n_{t}$ number of total alleles, when mixed with a balanced diploid population its theoretical BAF and LRR values are:

$$
\begin{aligned}
& B A F=\left|\frac{p \times n_{b}+1-p}{p \times n_{t}+2 \times(1-p)}-0.5\right|+x_{0} \\
& L R R=\log _{2}\left(p \times n_{t}+2 \times(1-p)\right)-1+y_{0}
\end{aligned}
$$

where $p$ is sAGP, and $\mathrm{x}_{0}, \mathrm{y}_{0}$ are the coordinates of the $\left(\mathrm{n}_{\mathrm{t}}=2, \mathrm{n}_{\mathrm{b}}=1\right)$ state. When $p$ changes, the points (BAF, LRR) follow a family of curved lines on the BAF-LRR plot, starting from the origin $\left(\mathrm{x}_{0}, \mathrm{y}_{0}\right)$. Each line corresponds to a unique combination of $\left(\mathrm{n}_{\mathrm{b}}, \mathrm{n}_{\mathrm{t}}\right)$ and is called a canonical line; and each point on this line uniquely corresponds to an sAGP value. The main task is to assign each observed segment to a canonical line. Due to noise, a sCNA does not locate precisely on a canonical line. Thus for each sCNA, we scan all possible canonical lines to find the one satisfying the following criteria:

a. Distance to the closest canonical line $\leq 2$ $\sqrt{s d_{B A F}^{2}+s d_{L R R}^{2}}$; where $s d^{2}{ }_{B A F}$ and $s d^{2}{ }_{L R R}$ are the estimated standard errors of BAF and LRR values.

Sometimes multiple lines satisfy (a) and result in multiple sAGP and $n_{t}$ estimates. In such cases we apply

b. Choose $s A G P=\operatorname{argmin}\left(F=n_{t}-\right.$ ploidy $\left.+\left|p_{s}-p\right|\right)$; where $\mathrm{p}_{\mathrm{s}}$ is sample-wide AGP and ploidy is the estimated global average ploidy from step ii). This criterion chooses the most probable canonical line as the one that results in a total copy number close to the genome-wide ploidy and an sAGP close to the global AGP.

If no canonical line can be found in (a), that is, the deviation is greater than the specified $2 \times$ scale of the standard deviations of BAF and LRR markers, we consider the sCNA not meeting the regional two-way mixing hypothesis, and its SAGP is assigned NA, its $n_{b}$ and $\mathrm{n}_{\mathrm{t}}$ are also treated as missing values in downstream analysis.

\section{Statistical modeling to infer macroscopic clonal structure}

As explained in the main text, sAGP values can be calculated for either predefined genomic bins or identified sCNAs. In the per-bin analysis, the user can choose to filter out the non-sCNA bins or those with very small sAGP values, as true sCNAs with length shorter than the bin width tend to have reduced sAGP estimates due to the flanking euploid regions. In our analysis of the breast tumor data we applied two filtering steps. First, we considered bins with median folded BAF $\leq 0.04$ and absolute median LRR $\leq 0.16$ to be euploid, and assigned sAGP $=0$. Second, before sAGP clustering, we removed bins with sAGP $\leq 0.05$ to remove the contribution of the small sAGP values. At this step there is an average of $\mathrm{n}=224$ bins left per sample. The two models described in the main text are evaluated in a maximal likelihood framework. For Model-1, the log likelihood has a uniform and a normal component:

$$
l=\sum_{i=1}^{n} \ln \left(\frac{A}{\operatorname{range}(\boldsymbol{Y})}+(1-A) \times \operatorname{Norm}\left(y_{i}, \mu, \sigma\right)\right)
$$

where $\mathbf{Y}$ is the observed sAGP vector for a given sample, with components $y_{i}, i=1,2, \ldots, n$, where $n$ is the number of DNA segments after filtering. A is a scalar so that A/ range provides the scaled uniform distribution. $\mu$ and $\sigma$ are the mean and standard deviation of the single peak in the model following the normal distribution. We constrain $\mathrm{A}$ and $\mu$ in the range $(0,1)$. The parameters $\mathrm{A}, \mu$, and $\sigma$ are estimated using the maximum likelihood approach, implemented in customized scripts (part of CHAT) written in the $\mathrm{R}$ statistical programming language [45].

Model-2 is fitted using a Dirichlet process Gaussian mixture model to infer the uncertain number of peaks and their relative abundances. The parameterization is as follows:

$$
\begin{aligned}
& y_{i} \mid \mu_{i}, \sigma_{i} \sim \operatorname{Norm}\left(\mu_{i}, \sigma_{i}\right), i=1,2 \ldots, n \\
& \mu_{i}, \sigma_{i} \mid G \sim G \\
& G \mid \alpha, G_{0} \sim \operatorname{DP}\left(\alpha G_{0}\right) \\
& G_{0}=\operatorname{Norm}\left(\mu \mid \mu_{1}, \sigma / k_{0}\right) \operatorname{InvWishart}\left(\sigma \mid v_{1}, \psi_{1}\right) \\
& G_{0}=\operatorname{Norm}\left(\mu \mid \mu_{1}, \sigma / k_{0}\right) \operatorname{InvWishart}\left(\sigma \mid v_{1}, \psi_{1}\right) \\
& k_{0} \mid \tau_{1}, \tau_{2} \sim \Gamma\left(\tau_{1} / 2, \tau_{2} / 2\right)
\end{aligned}
$$

Together these expressions describe a standard Dirichlet process mixture of normal model [40]. The implementation of the MCMC fitting is via R package DPpackage [56]. There are different ways to specify the prior parameters for the normal mixture model. The baseline Gaussian distribution $G_{0}$ relies on three prior parameters, $\mu_{1}, \sigma$, and $k_{0}$, where $\sigma$ is explicitly modeled by an Inversed Wishart distribution with priors $v_{1}$ and $\psi_{1}$, and $k_{0}$ follows a Gamma distribution. In practice, the hyperpriors, $v_{1}, \psi_{1}$, and $\mathrm{k}_{0}$ can also be allowed to be random variables with a given prior distribution, and the model will have higher power to fit minor peaks in the data. In this work we used 
a conservative setting of prior parameters in terms of peak discovery sensitivity.

Model-1 cannot be included as a special case of Model-2, since when $y$ is truly uniformly distributed, Dirichlet process tends to call multiple peaks instead of one peak, even with current conservative prior setting. Our solution is to fit both models, then numerically compute the likelihood of each model, and use Bayesian Information Criterion (BIC) to select the better model. Model-1 has three free parameters: $\mathrm{A}, \mu$, and $\sigma$, while Model-2 has seven: $a_{0}$, $b_{0}, k_{0}, v_{1}, \psi_{1}, \tau_{1}$, and $\tau_{2}$.

\section{CCF estimation and scenario identifiability for CN-LOH and deletion}

In the main text we presented how CHAT performs CCF estimation for the case of hemizygous amplifications $\left(\mathrm{n}_{\mathrm{b}}=1, \mathrm{n}_{\mathrm{t}}=3\right)$. While this sCNA type has all four scenarios, Scenario $\mathbf{B}$ is not available for some other types of sCNAs, including $\mathrm{LOH}\left(\mathrm{n}_{\mathrm{b}}=0, \mathrm{n}_{\mathrm{t}} \geq 1\right)$ and balanced allelic gains $\left(n_{b}>1, n_{t}=2 n_{b}\right)$. Below we will describe the cases of copy neutral LOH $(\mathrm{CN}-\mathrm{LOH})$ and hemizygous deletion.

\section{$\mathrm{CN}-\mathrm{LOH}$}

In Scenario $\mathbf{A}_{\mathbf{1}}$ (Additional file 1: Figure S1A)

$$
\begin{aligned}
& r_{0}+r_{1}+r_{2}=1 \\
& r_{2}=p \\
& f=\frac{r_{1}+n_{a} \times r_{2}}{n_{t}}=\frac{r_{1}+2 \times r_{2}}{2}
\end{aligned}
$$

where $n_{t}=2 \times(1-p)+n_{t} \times p=2$ and $C C F=r_{1}+r_{2}$. Using the above equations it is easy to show that

$$
C C F^{A_{1}}\left(f, n_{b}, n_{t}, p\right)=n_{t} \times f-p \times n_{a}+p=2 \times f-p
$$

Scenarios $\mathbf{A}_{2}, \mathbf{B}$, and $\mathbf{C}$ have the same expression:

$$
C C F^{A_{2}}=C C F^{B}=C C F^{C}=2 f
$$

Note that $\mathbf{A}_{2}$ and $\mathbf{C}$ not only have the same expression for CCF, they also have the same three-population composition, although the three populations emerge by different evolutionary routes. A previous study [32] failed to take $\mathbf{A}_{\mathbf{1}}$ into consideration and could have overestimated CCF under $\mathbf{A}_{1}$, thus could have designated subclonal mutations as clonal when $\mathbf{A}_{\mathbf{1}}$ is the true evolutionary scenario.

The lower and upper limits of SAF in each scenario can be derived using the same process as in the main text. In scenario $\mathbf{A}_{\mathbf{1}}, f$ reaches its upper limit when $\mathrm{r}_{0}=0$, and $r_{1}=1-r_{2}$.

$$
f_{h}^{A_{1}}=\frac{1-p+2 \times p}{n_{t}}=\frac{1+p}{2}
$$

On the opposite side is $r_{1}$ is zero, when $f$ reaches its minimum value:

$$
f_{l}^{A_{1}}=\frac{2 p}{2}=p
$$

With $p$ takes values from $(0,1)$, the areas defined by these limits are shown in Figure 4B.

For scenario B:

$$
\begin{aligned}
& f_{h}^{B}=\frac{p}{N_{t}}=\frac{p}{2} \\
& f_{l}^{B}=0
\end{aligned}
$$

And scenario $\mathbf{C}$ :

$$
\begin{aligned}
& f_{h}^{C}=\frac{1-p}{N_{t}}=\frac{1-p}{2} \\
& f_{l}^{C}=0
\end{aligned}
$$

\section{Hemizygous deletion}

All four scenarios have the same expression:

$$
C C F^{A_{1}}=C C F^{A_{2}}=C C F^{B}=C C F^{C}=f(2-p)
$$

Similar to $\mathrm{CN}-\mathrm{LOH}, \mathbf{A}_{2}$ and $\mathbf{C}$ not only have the same expression for CCF, they also have the same threepopulation composition.

The upper and lower limits for this sCNA type are:

$$
\begin{aligned}
f_{h}^{A_{1}} & =\frac{1-p+p}{N_{t}}=\frac{1}{2-p} \\
f_{l}^{A_{1}} & =\frac{p}{2-p} \\
f_{h}^{B} & =\frac{p}{N_{t}}=\frac{p}{2-p} \\
f_{l}^{B} & =0 \\
f_{h}^{C} & =\frac{1-p}{N_{t}}=\frac{1-p}{2-p} \\
f_{l}^{C} & =0
\end{aligned}
$$

The areas defined by these limits are shown in Figure 4C. 


\section{Variance of CCF}

We use the same approach as described in [32] to estimate the standard deviation of CCF. The distribution of CCF is modeled as Binomial:

$$
\operatorname{Pr}(C C F=x) \propto \operatorname{Binomi}(S \mid N, G(x, p, \Theta))
$$

where $S$ is the read count for the somatic allele and $\mathrm{N}$ is the total read depth. $G(\bullet)$ is expected value of SAF given CCF value $x$, sAGP value $p$, and lineage scenario $\Theta$. $G$ is simply obtained by reversing the CCF expressions described in the main text (Equations (1), (2), (3), (4), (5), (6), and (7)). We assume a uniform prior on $x$ and the expectation and variance of CCF can be calculated as:

$$
\begin{aligned}
& \operatorname{EXP}(C C F)=\frac{\int_{0}^{1} \operatorname{Binom}(S \mid N, G) x d x}{\int_{0}^{1} \operatorname{Binom}(S \mid N, G) d x} \\
& \operatorname{Var}(C C F)=\frac{\int_{0}^{1} \operatorname{Binom}(S \mid N, G) x^{2} d x}{\int_{0}^{1} \operatorname{Binom}(S \mid N, G) d x-E X P(C C F)^{2}}
\end{aligned}
$$

To note, the expectations of CCF are identical to the expressions in the main text (Equations (1), (2), (3), (4), (5), (6), and (7)).

\section{Probabilistic scenario identification}

The task is to use the observed somatic allele frequency $(f)$ and sAGP value to determine the most likely scenario among the four scenarios described in the main text. We assume that $\mathrm{f}$ has a uniform prior, $\mathrm{U}(0,1)$, and we are interested in calculating the likelihood that the SSNV occurred before the sCNA, given the copy number configuration $\left(\mathrm{n}_{\mathrm{b}}, \mathrm{n}_{\mathrm{t}}\right)$, known sAGP $(\mathrm{p})$, and the observed allele counts. Let $f_{0}$ denote the true $f$. The probability of observing $\mathrm{S}$ count of the somatic allele is model by Bino$\operatorname{mial}\left(\mathrm{f}_{0}, \mathrm{~N}\right)$ and the likelihood of each scenario is the probability of observing $\mathrm{S}$ given the scenario is true, integrated over all the possible values of $\mathrm{f}_{0}$ :

$$
\begin{aligned}
p_{X} & =L\left(\text { Scenario } X \mid p, n_{b}, n_{t}, N, S\right) \\
& =\operatorname{Pr}\left\{S \mid X, p, n_{b}, n_{t}, N\right\} \\
& =\int \operatorname{Pr}\left\{S \mid f_{0}, N\right\} \times \operatorname{Pr}\left\{f=f_{0} \mid X, p, n_{b}, n_{t}\right\} \mathrm{d} f_{0} \\
& =\int_{f_{l}^{X}}^{f_{h}^{X}} \operatorname{Pr}\left\{S \mid f_{0}, N\right\} d f_{0}
\end{aligned}
$$

where $\mathrm{X}$ is $\mathbf{A}_{\mathbf{1}}, \mathbf{A}_{2} \mathrm{C}$ scenarios, and $\mathrm{f}_{\mathrm{h}}^{\mathrm{X}}, \mathrm{f}_{\mathrm{l}}^{\mathrm{X}}$ are computed according to Equations (8), (9), (10), (11), (12), (13), (14), and (15) in the main text and those in the section (CCF estimation and scenario identifiability for $\mathrm{CN}-\mathrm{LOH}$ and deletion) above. We then compute the summation of $\mathrm{p}_{\mathrm{X}}$ :

$$
P=p_{A 1}+p_{A 2}+p_{B}+p_{C}
$$

and normalize each likelihood using $P$ :

$$
\tilde{p}_{X}=\frac{p_{X}}{P}
$$

We calculate the normalized probability for each scenario, as well as all the possible combinations of multiple scenarios. For example, the probability of either scenario $\mathrm{A} 1$ or $\mathrm{C}$ is $\tilde{p}_{A 1 C}=\tilde{p}_{A 1}+\tilde{p}_{C}$. There are in total $2^{4}-1=15$ possible combinations. If the normalized probability of any of the four scenarios is greater than 0.95 , the SNV is assigned to the corresponding scenario. If none of the single-scenario probability exceeds 0.95 , we ask if any of the six two-scenario combinations have probability $>0.95$. If this step fails, we next examine the four possible three-scenario combinations, and so forth. If all the above steps fail, we report the SNV scenario $\mathbf{A}_{1} / \mathbf{A}_{2} / \mathbf{B} / \mathbf{C}$, and no unique CCF can be estimated in this case.

\section{In silico validation and computation performance Performance of SAGP inference}

We first tested the performance of CHAT in sAGP estimation. We simulated LRR and BAF values for a series of sCNA datasets with two aneuploid tumor populations, which are mixed with the euploid population. The first population is the dominant clone, with an assigned sAGP value of $\mathrm{p}_{\mathrm{dom}} \sim[0.1,0.2, \ldots, 1.0]$. The second population is a minor clone, with an assigned sAGP value of $\mathrm{p}_{\mathrm{sub}} \sim\left[0,0.1, \ldots \mathrm{p}_{\mathrm{dom}}-0.1\right]$. The fraction of the euploid population is $1-\mathrm{p}_{\text {dom }}-\mathrm{p}_{\text {sub }}$. In all, there are $55 \mathrm{p}_{\text {dom }}-\mathrm{p}_{\text {sub }}$ combinations; and for each, we simulated 200 euploid segments $\left(\mathrm{n}_{\mathrm{b}}=1, \mathrm{n}_{\mathrm{t}}=2\right.$, sAGP $\left.=0\right)$ and $200 \mathrm{sCNA}$ segments, of which 133 (about 2/3) were assigned to the dominant clone $\left(\mathrm{sAGP}=\mathrm{p}_{\mathrm{dom}}\right)$, and the remaining 67 were assigned to the minor clone $\left(\mathrm{sAGP}=\mathrm{p}_{\text {sub }}\right)$. Within each clone, the sCNAs were assigned to one of four copy number configurations with the following ratios: $2 / 7$ for deletion $\left(n_{b}=0\right.$, $\left.\mathrm{n}_{\mathrm{t}}=1\right), 2 / 7$ for CN-LOH $\left(\mathrm{n}_{\mathrm{b}}=0, \mathrm{n}_{\mathrm{t}}=2\right), 2 / 7$ for amplification $\left(\mathrm{n}_{\mathrm{b}}=1, \mathrm{n}_{\mathrm{t}}=3\right)$, and $1 / 7$ for balanced doubling $\left(\mathrm{n}_{\mathrm{b}}=2\right.$, $n_{t}=4$ ). The BAF and LRR values were generated using the assigned sAGP and copy number configuration with the following formula:

$$
\begin{aligned}
& B A F=\left|0.5-\frac{p \times n_{b}+1-p}{n_{t}}\right|+\operatorname{Normal}\left(0, \sigma_{B A F}\right) \\
& L R R=\log _{2} n_{t}-1+\operatorname{Normal}\left(0, \sigma_{L R R}\right)
\end{aligned}
$$

where $\mathrm{p}$ stands for sAGP, and $\mathrm{n}_{\mathrm{t}}$ is the averaged total copy number for the local segment: $2(1-\mathrm{p})+\mathrm{n}_{\mathrm{t}} \times \mathrm{p}$. $\sigma_{\mathrm{BAF}}$ 
and $\sigma_{\text {LRR }}$ are the standard deviation values of the persegment BAF and LRR, respectively. For the Affymetrix 6.0 platform, the per-SNP standard deviation for BAF is about 0.05 , and for LRR is about 0.25 (our observation). Thus the choice of $\sigma_{\mathrm{BAF}}=0.01$ and $\sigma_{\mathrm{LRR}}=0.04$ is equivalent to the standard error of an sCNA of approximately 36 SNP markers. For a 1 million SNP platform, 36 SNPs cover approximately $110 \mathrm{~kb}$, therefore ours are conservative choices for sCNAs $110 \mathrm{~kb}$ or longer, profiled by 1 million SNPs or more.

After generating the BAF and LRR values using Equations (16) and (17) for the 400 segments for each of the $55 \mathrm{p}_{\mathrm{dom}}-\mathrm{p}_{\text {sub }}$ combinations, we applied CHAT to estimate sAGP, $n_{b}$, and $n_{t}$ for each simulated segment and reported the results in Figure 5A.

\section{Performance of CCF estimation}

Of the $55 \mathrm{p}_{\mathrm{dom}}-\mathrm{p}_{\text {sum }}$ combinations described above we selectively tested CCF inference in four cases: $\mathrm{p}_{\text {dom }}$ $\mathrm{p}_{\text {sum }} \sim(0.9,0.8),(0.9,0.4),(0.5,0.3)$, and $(0.3,0.1)$. For each case, we simulated 4,000 SNVs, of which approximately 2,000 fall in the 200 euploid segments, and the other approximately 2,000 fall in the $200 \mathrm{sCNA}$ regions, with the $\left(\mathrm{sAGP}, \mathrm{n}_{\mathrm{b}}, \mathrm{n}_{\mathrm{t}}\right.$ ) assignment implemented as described above. In effect we assume that the euploid intervals account for $50 \%$ of the genome. To make the test realistic, we used the sAGP, $\mathrm{n}_{\mathrm{b}}$, and $\mathrm{n}_{\mathrm{t}}$ estimated by CHAT rather than the true values used in the initial simulation of the LRR and BAF data. If the SNV falls in a euploid region, the assigned SAF was randomly drawn from uniform $(0,0.5)$ and the corresponding 'true' $\mathrm{CCF}=\mathrm{SAF} \times 2$. If it falls in an aneuploid region, we randomly choose the lineage scenario from $\left(\mathbf{A}_{1}, \mathbf{A}_{2}, \mathbf{B}, \mathbf{C}\right)$ according to the local copy number configuration. If the sCNA is a $\mathrm{CN}-\mathrm{LOH}$ or balanced doubling region, we limit the scenarios to $\left(\mathbf{A}_{\mathbf{1}}, \mathbf{B}, \mathbf{C}\right)$. The upper and lower limits of the chosen scenario were determined using Equations (8) to (15) in the main text and the equations in Materials and methods, CCF estimation and scenario identifiability for $\mathrm{CN}-\mathrm{LOH}$ and deletion. SAF values were then randomly drawn from within this permissible range: uniform $\left(f_{l}, f_{h}\right)$, where $f_{l}$ and $f_{h}$ were the lower and upper limits. 'True' CCF values were computed using Equations (1) to (7) in the main text. Lastly, from the 'true' CCF we simulated the allele counts in two steps. For a mean read depth $k$, the actual coverage at a given $\mathrm{SNV}, \mathrm{N}$, was sampled from $\mathrm{N} \sim \operatorname{Poisson}(k)$. With $\mathrm{N}$ and $\mathrm{f}$ (that is, CCF) thus assigned, the count of the somatic mutation allele was sampled from $\operatorname{Binomial}(f, \mathrm{~N})$. Based on the estimated sAGP, copy number configuration and the simulated somatic allele counts we used CHAT to estimate CCF. The estimated values were compared with the 'true' CCF for both $k=50$ and $k=100$. For all eight cases (four $\mathrm{p}_{\mathrm{dom}}-\mathrm{p}_{\mathrm{sub}}$ combinations and two $\mathrm{k}$ values) we calculated the Spearman's rank correlation coefficient and/or the median absolute deviation (MAD) between the known and estimated CCF values (Figure 5B and C).

\section{Computational requirements}

We estimated the time and memory requirement of CHAT using the TCGA dataset for breast tumors. The time estimate below is based on allele-specific copy number data with $850 \mathrm{~K}$ SNPs for tumor-normal pairs and whole-exome sequencing data with approximately $30 \times$ average coverage. For binned segmentation (approximately 500 heterozygous SNPs per bin), it takes 2 min to complete the sAGP and CCF estimation for one tumor/normal pair, and it requires about $10 \mathrm{MB}$ memory. For detected sCNAs, the computational time increases to an average of 12 min per sample pair. The above estimation is based on running $R$ scripts with a single processor (AMD Opteron 6136, 2.4GHz with 4G RAM) and counting input file reading time. In CHAT, the user can apply the $\mathrm{R}$ package parallel to enable multi-thread processing. This allows the use of as many processors as available. On our server (32 AMD Opteron 6136 CPUs and 128G RAM), our test run used 14 processors on average, and it took $10 \mathrm{~h}$ (140 CPU-hours) to complete the CBS segmentation, sAGP estimation for 732 breast tumor-normal samples and CCF estimation for 445 samples with downloaded VCF files.

\section{Comparison with EXPANDS and PyClone}

We simulated 200 CNA regions, with sAGP values randomly drawn from $\mathrm{U}(0,1)$, and copy number configurations assigned by the ratio of $2 / 7$ for deletion, $2 / 7$ for $\mathrm{CN}-\mathrm{LOH}$, $2 / 7$ for amplification, and 1/7 for balanced doubling, as described in Performance of sAGP inference. For each CNA, the LRR and BAF values were simulated as in Performance of sAGP inference. We then simulated 1,000 somatic mutations evenly across the 'genome', with 488 that happened to fall in an sCNA region (with the rest falling in euploid regions). For these 488 somatic mutations, we assigned them to lineage scenarios with similar ratios across $A_{1}$, $\mathrm{A}_{2}$-when possible, $\mathrm{B}$, and $\mathrm{C}$. The actual number assigned to each combination was shown in Figure 6D. For each mutation thus assigned, we sampled the somatic allele frequency (f) uniformly from its permissible range as described in Performance of CCF estimation, calculate the corresponding true CCF value, and simulated the sequencing read counts at the average coverage of $\mathrm{k}=50$, as described in Performance of CCF estimation. We applied CHAT to estimate (sAGP, $\mathrm{n}_{\mathrm{t}}, \mathrm{n}_{\mathrm{b}}$ ) from the simulated LRR and BAF data, then estimated CCF from the simulated read counts, sAGP and the estimated lineage scenarios and $\left(\mathrm{n}_{\mathrm{t}}, \mathrm{n}_{\mathrm{b}}\right)$ status. In parallel, we applied PyClone to the same dataset, using the simulated read counts and the 
true $\left(\mathrm{n}_{\mathrm{t}}, \mathrm{n}_{\mathrm{b}}\right)$ as input to estimate CCF. The choice of true $\left(\mathrm{n}_{\mathrm{t}}, \mathrm{n}_{\mathrm{b}}\right)$ rather than the CHAT-estimated $\left(\mathrm{n}_{\mathrm{t}}, \mathrm{n}_{\mathrm{b}}\right)$ should slightly favor PyClone as the errors in estimating $\left(\mathrm{n}_{\mathrm{t}}, \mathrm{n}_{\mathrm{b}}\right)$ are not incorporated. Lastly, we applied EXPANDS to estimate CCF, using the simulated LRR and the observed somatic allele frequency as input. We compared the estimated CCF of the three tools with the known CCF in Figure 6. To make Figure 6D less cluttered we omitted balanced amplifications, thus only showing 423 mutations for the other CNA types.

\section{Data availability}

CHAT source package is available at https://sourceforge. net/projects/clonalhetanalysistool/files/?. It is released under fully open source license, GPL $(\geq 2.0)$. It is also available as a CRAN-R package. The breast tumor data were downloaded from the Cancer Genome Atlas Data Portal as described in Data access and sCNA identification. The TCGA IDs for the 732 tumors are in Additional file 2. The simulated data are available at http://sourceforge. net/projects/clonalhetanalysistool/files/simulations/ and as Additional file 3.

\section{Additional files}

\section{Additional file 1: Supplementary figures (Figure S1-S3) and legends describing additional information. \\ Additional file 2: TCGA Sample IDs for the $\mathbf{7 3 2}$ breast tumors we analyzed in this study. \\ Additional file 3: Simulated data used to compare CHAT, PyClone, and EXPANDS.}

\section{Abbreviations}

CCF: Cancer cell fraction; CHAT: Clonal heterogeneity analysis tool; ICGC: International Cancer Genome Consortium; SAF: Somatic allele frequency; sAGP: Segmental aneuploidy genome proportion; sCNA: Somatic copy number alteration; TCGA: The Cancer Genome Atlas.

\section{Competing interests}

The authors declare that they have no competing interests.

\section{Authors' contributions}

$B L$ developed and implemented the algorithm; $B L$ and $J L$ wrote the manuscript; JL supervised the study. Both authors read and approved the manuscript.

\section{Acknowledgments}

The results presented here are in part based upon data generated by the TCGA Research Network: http://cancergenome.nih.gov/. We would like to thank a Rackham Predoctoral Fellowship (BL) and a Pilot Grant from the Center of Computation Biology and Medicine at University of Michigan ( $J$ ) for supporting this research. We thank Drs. Nancy Zhang, Kerby Shedden, and Sebastian Zoellner for helpful discussions.

\section{Author details}

${ }^{1}$ Program of Bioinformatics, University of Michigan, 5940A Buhl, Box 5618, Ann Arbor, Ml 48109-5618, USA. ${ }^{2}$ Department of Human Genetics, University of Michigan, 5940A Buhl, Box 5618, Ann Arbor, Ml 48109-5618, USA.
Received: 31 July 2014 Accepted: 15 September 2014

Published online: 25 September 2014

\section{References}

1. Nowell PC: The clonal evolution of tumor cell populations. Science 1976, 194:23-28.

2. Fidler IJ: Tumor heterogeneity and the biology of cancer invasion and metastasis. Cancer Res 1978, 38:2651-2660.

3. Knudson AG Jr: Mutation and cancer: statistical study of retinoblastoma. Proc Natl Acad Sci U S A 1971, 68:820-823.

4. Fearon $E R$, Vogelstein B: A genetic model for colorectal tumorigenesis. Cell 1990, 61:759-767.

5. Vogelstein B, Kinzler KW: The multistep nature of cancer. Trends Genet 1993, 9:138-141.

6. Sjoblom T, Jones S, Wood LD, Parsons DW, Lin J, Barber TD, Mandelker D, Leary RJ, Ptak J, Silliman N, Szabo S, Buckhaults P, Farrell C, Meeh P, Markowitz SD, Willis J, Dawson D, Willson JK, Gazdar AF, Hartigan J, Wu L, Liu C, Parmigiani G, Park BH, Bachman KE, Papadopoulos N, Vogelstein B, Kinzler KW, Velculescu VE: The consensus coding sequences of human breast and colorectal cancers. Science 2006, 314:268-274.

7. Wood LD, Parsons DW, Jones S, Lin J, Sjoblom T, Leary RJ, Shen D, Boca SM, Barber T, Ptak J, Silliman N, Szabo S, Dezso Z, Ustyanksky V, Nikolskaya T, Nikolsky Y, Karchin R, Wilson PA, Kaminker JS, Zhang Z, Croshaw R, Willis J, Dawson D, Shipitsin M, Willson JK, Sukumar S, Polyak K, Park BH, Pethiyagoda CL, Pant PV, et al: The genomic landscapes of human breast and colorectal cancers. Science 2007, 318:1108-1113.

8. Parsons DW, Jones S, Zhang X, Lin JC, Leary RJ, Angenendt P, Mankoo P, Carter H, Siu IM, Gallia GL, Olivi A, McLendon R, Rasheed BA, Keir S, Nikolskaya T, Nikolsky Y, Busam DA, Tekleab H, Diaz LA Jr, Hartigan J, Smith DR, Strausberg RL, Marie SK, Shinjo SM, Yan H, Riggins GJ, Bigner DD, Karchin R, Papadopoulos N, Parmigiani G, et al: An integrated genomic analysis of human glioblastoma multiforme. Science 2008, 321:1807-1812.

9. Jones S, Zhang X, Parsons DW, Lin JC, Leary RJ, Angenendt P, Mankoo P, Carter H, Kamiyama H, Jimeno A, Hong SM, Fu B, Lin MT, Calhoun ES, Kamiyama M, Walter K, Nikolskaya T, Nikolsky Y, Hartigan J, Smith DR, Hidalgo M, Leach SD, Klein AP, Jaffee EM, Goggins M, Maitra A, lacobuzio-Donahue C, Eshleman JR, Kern SE, Hruban RH, et al: Core signaling pathways in human pancreatic cancers revealed by global genomic analyses. Science 2008, 321:1801-1806.

10. Greaves M, Maley CC: Clonal evolution in cancer. Nature 2012, 481:306-313.

11. Yates $L R$, Campbell PJ: Evolution of the cancer genome. Nat Rev Genet 2012, 13:795-806.

12. The Cancer Genome Atlas Research Network: Comprehensive genomic characterization defines human glioblastoma genes and core pathways. Nature 2008, 455:1061-1068.

13. The Cancer Genome Atlas Research Network: Integrated genomic analyses of ovarian carcinoma. Nature 2011, 474:609-615.

14. The Cancer Genome Atlas Research Network: Comprehensive molecular portraits of human breast tumours. Nature 2012, 490:61-70.

15. The Cancer Genome Atlas Research Network: Comprehensive genomic characterization of squamous cell lung cancers. Nature 2012, 489:519-525.

16. Alexandrov LB, Nik-Zainal S, Wedge DC, Aparicio SA, Behjati S, Biankin AV, Bignell GR, Bolli N, Borg A, Borresen-Dale AL, Boyault S, Burkhardt B, Butler AP, Caldas C, Davies HR, Desmedt C, Eils R, Eyfjord JE, Foekens JA, Greaves M, Hosoda F, Hutter B, llicic T, Imbeaud S, Imielinski M, Jager N, Jones DT, Jones D, Knappskog S, Kool M, et al: Signatures of mutational processes in human cancer. Nature 2013, 500:415-421.

17. Van Loo P, Nordgard SH, Lingjaerde OC, Russnes HG, Rye $\mathrm{H}_{\text {, Sun }}$ W Weigman VJ, Marynen P, Zetterberg A, Naume B, Perou CM, Borresen-Dale $\mathrm{AL}$, Kristensen VN: Allele-specific copy number analysis of tumors. Proc Natl Acad Sci U S A 2010, 107:16910-16915.

18. Li B, Senbabaoglu Y, Peng W, Yang ML, Xu J, Li JZ: Genomic estimates of aneuploid content in glioblastoma multiforme and improved classification. Clin Cancer Res 2012, 18:5595-5605.

19. Popova T, Manie E, Stoppa-Lyonnet D, Rigaill G, Barillot E, Stern MH: Genome Alteration Print (GAP): a tool to visualize and mine complex cancer genomic profiles obtained by SNP arrays. Genome Bio/ 2009, 10:R128.

20. Sturm D, Witt H, Hovestadt V, Khuong-Quang DA, Jones DT, Konermann C, Pfaff E, Tonjes M, Sill M, Bender S, Kool M, Zapatka M, Becker N, Zucknick M, Hielscher T, Liu XY, Fontebasso AM, Ryzhova M, Albrecht S, Jacob K, Wolter 
M, Ebinger M, Schuhmann MU, van Meter T, Fruhwald MC, Hauch H, Pekrun A, Radlwimmer B, Niehues T, von Komorowski G, et al: Hotspot mutations in $\mathrm{H} 3 \mathrm{~F} 3 \mathrm{~A}$ and IDH1 define distinct epigenetic and biological subgroups of glioblastoma. Cancer Cell 2012, 22:425-437.

21. Lawrence MS, Stojanov P, Polak P, Kryukov GV, Cibulskis K, Sivachenko A, Carter SL, Stewart C, Mermel CH, Roberts SA, Kiezun A, Hammerman PS, McKenna A, Drier Y, Zou L, Ramos AH, Pugh TJ, Stransky N, Helman E, Kim J, Sougnez C, Ambrogio L, Nickerson E, Shefler E, Cortes ML, Auclair D, Saksena G, Voet D, Noble M, DiCara D, et al: Mutational heterogeneity in cancer and the search for new cancer-associated genes. Nature 2013, 499:214-218.

22. Verhaak RG, Hoadley KA, Purdom E, Wang V, Qi Y, Wilkerson MD, Miller CR, Ding L, Golub T, Mesirov JP, Alexe G, Lawrence M, O'Kelly M, Tamayo P, Weir BA, Gabriel S, Winckler W, Gupta S, Jakkula L, Feiler HS, Hodgson JG, James CD, Sarkaria JN, Brennan C, Kahn A, Spellman PT, Wilson RK, Speed TP, Gray JW, Meyerson M, et al: Integrated genomic analysis identifies clinically relevant subtypes of glioblastoma characterized by abnormalities in PDGFRA, IDH1, EGFR, and NF1. Cancer Cell 2010, 17:98-110.

23. Curtis C, Shah SP, Chin SF, Turashvili G, Rueda OM, Dunning MJ, Speed D, Lynch AG, Samarajiwa S, Yuan Y, Graf S, Ha G, Haffari G, Bashashati A, Russell R, McKinney S, Group M, Langerod A, Green A, Provenzano E, Wishart G, Pinder S, Watson P, Markowetz F, Murphy L, Ellis I, Purushotham A, Borresen-Dale AL, Brenton JD, Tavare $S$, et al: The genomic and transcriptomic architecture of 2,000 breast tumours reveals novel subgroups. Nature 2012, 486:346-352.

24. Garraway LA, Lander ES: Lessons from the cancer genome. Cell 2013, 153:17-37.

25. Navin N, Kendall J, Troge J, Andrews P, Rodgers L, Mclndoo J, Cook K, Stepansky A, Levy D, Esposito D, Muthuswamy L, Krasnitz A, McCombie WR, Hicks J, Wigler M: Tumour evolution inferred by single-cell sequencing. Nature 2011, 472:90-94.

26. Shalek AK, Satija R, Adiconis X, Gertner RS, Gaublomme JT, Raychowdhury R, Schwartz S, Yosef N, Malboeuf C, Lu D, Trombetta JJ, Gennert D, Gnirke A, Goren A, Hacohen N, Levin JZ, Park H, Regev A: Single-cell transcriptomics reveals bimodality in expression and splicing in immune cells. Nature 2013, 498:236-240.

27. Hou Y, Song L, Zhu P, Zhang B, Tao Y, Xu X, Li F, Wu K, Liang J, Shao D, Wu H, Ye X, Ye C, Wu R, Jian M, Chen Y, Xie W, Zhang R, Chen L, Liu X, Yao X, Zheng H, Yu C, Li Q, Gong Z, Mao M, Yang X, Yang L, Li J, Wang W, et al: Single-cell exome sequencing and monoclonal evolution of a JAK2-negative myeloproliferative neoplasm. Cell 2012, 148:873-885.

28. $X u X$, Hou Y, Yin X, Bao L, Tang A, Song L, Li F, Tsang S, Wu K, Wu H, He W, Zeng L, Xing M, Wu R, Jiang H, Liu X, Cao D, Guo G, Hu X, Gui Y, Li Z, Xie W, Sun X, Shi M, Cai Z, Wang B, Zhong M, Li J, Lu Z, Gu N, et al: Single-cell exome sequencing reveals single-nucleotide mutation characteristics of a kidney tumor. Cell 2012, 148:886-895.

29. Keats JJ, Chesi M, Egan JB, Garbitt VM, Palmer SE, Braggio E, Van Wier S, Blackburn PR, Baker AS, Dispenzieri A, Kumar S, Rajkumar SV, Carpten JD, Barrett M, Fonseca R, Stewart AK, Bergsagel PL: Clonal competition with alternating dominance in multiple myeloma. Blood 2012, 120:1067-1076.

30. Ley TJ, Ding L, Walter MJ, McLellan MD, Lamprecht T, Larson DE, Kandoth C, Payton JE, Baty J, Welch J, Harris CC, Lichti CF, Townsend RR, Fulton RS, Dooling DJ, Koboldt DC, Schmidt H, Zhang Q, Osborne JR, Lin L, O'Laughlin M, McMichael JF, Delehaunty KD, McGrath SD, Fulton LA, Magrini VJ, Vickery TL, Hundal J, Cook LL, Conyers JJ, et al: DNMT3A mutations in acute myeloid leukemia. N Engl J Med 2010, 363:2424-2433.

31. Durinck S, Ho C, Wang NJ, Liao W, Jakkula LR, Collisson EA, Pons J, Chan SW, Lam ET, Chu C, Park K, Hong SW, Hur JS, Huh N, Neuhaus IM, Yu SS, Grekin RC, Mauro TM, Cleaver JE, Kwok PY, LeBoit PE, Getz G, Cibulskis K, Aster JC, Huang H, Purdom E, Li J, Bolund L, Arron ST, Gray JW, et al: Temporal dissection of tumorigenesis in primary cancers. Cancer Discov 2011, 1:137-143

32. Landau DA, Carter SL, Stojanov P, McKenna A, Stevenson K, Lawrence MS, Sougnez C, Stewart C, Sivachenko A, Wang L, Wan Y, Zhang W, Shukla SA, Vartanov A, Fernandes SM, Saksena G, Cibulskis K, Tesar B, Gabriel S, Hacohen N, Meyerson M, Lander ES, Neuberg D, Brown JR, Getz G, Wu CJ: Evolution and impact of subclonal mutations in chronic lymphocytic leukemia. Cell 2013, 152:714-726.

33. Gerlinger M, Rowan AJ, Horswell S, Larkin J, Endesfelder D, Gronroos E, Martinez P, Matthews N, Stewart A, Tarpey P, Varela I, Phillimore B, Begum S,
McDonald NQ, Butler A, Jones D, Raine K, Latimer C, Santos CR, Nohadani M, Eklund AC, Spencer-Dene B, Clark G, Pickering L, Stamp G, Gore M, Szallasi Z, Downward J, Futreal PA, Swanton C: Intratumor heterogeneity and branched evolution revealed by multiregion sequencing. N Engl J Med 2012, 366:883-892.

34. Sottoriva A, Spiteri I, Piccirillo SG, Touloumis A, Collins VP, Marioni JC, Curtis C, Watts C, Tavare S: Intratumor heterogeneity in human glioblastoma reflects cancer evolutionary dynamics. Proc Natl Acad Sci U S A 2013, 110:4009-4014.

35. Yachida S, Jones S, Bozic I, Antal T, Leary R, Fu B, Kamiyama M, Hruban RH, Eshleman JR, Nowak MA, Velculescu VE, Kinzler KW, Vogelstein B, lacobuzio-Donahue CA: Distant metastasis occurs late during the genetic evolution of pancreatic cancer. Nature 2010, 467:1114-1117.

36. Campbell PJ, Yachida S, Mudie $L$, Stephens PJ, Pleasance ED, Stebbings LA, Morsberger LA, Latimer C, McLaren S, Lin ML, McBride DJ, Varela I, Nik-Zainal SA, Leroy C, Jia M, Menzies A, Butler AP, Teague JW, Griffin CA, Burton J, Swerdlow H, Quail MA, Stratton MR, lacobuzio-Donahue C, Futreal PA: The patterns and dynamics of genomic instability in metastatic pancreatic cancer. Nature 2010, 467:1109-1113.

37. McFadden DG, Papagiannakopoulos T, Taylor-Weiner A, Stewart C, Carter SL, Cibulskis K, Bhutkar A, McKenna A, Dooley A, Vernon A, Sougnez C, Malstrom S, Heimann M, Park J, Chen F, Farago AF, Dayton T, Shefler E, Gabriel S, Getz G, Jacks T: Genetic and clonal dissection of murine small cell lung carcinoma progression by genome sequencing. Cell 2014, 156:1298-1311.

38. Nik-Zainal S, Van Loo P, Wedge DC, Alexandrov LB, Greenman CD, Lau KW, Raine K, Jones D, Marshall J, Ramakrishna M, Shlien A, Cooke SL, Hinton J, Menzies A, Stebbings LA, Leroy C, Jia M, Rance R, Mudie LJ, Gamble SJ, Stephens PJ, McLaren S, Tarpey PS, Papaemmanuil E, Davies HR, Varela I, McBride DJ, Bignell GR, Leung K, Butler AP, et al: The life history of 21 breast cancers. Cell 2012, 149:994-1007.

39. Shah SP, Roth A, Goya R, Oloumi A, Ha G, Zhao Y, Turashvili G, Ding J, Tse K, Haffari G, Bashashati A, Prentice LM, Khattra J, Burleigh A, Yap D, Bernard V, McPherson A, Shumansky K, Crisan A, Giuliany R, Heravi-Moussavi A, Rosner J, Lai D, Birol I, Varhol R, Tam A, Dhalla N, Zeng T, Ma K, Chan SK, et al: The clonal and mutational evolution spectrum of primary triple-negative breast cancers. Nature 2012, 486:395-399.

40. Carter SL, Cibulskis K, Helman E, McKenna A, Shen H, Zack T, Laird PW, Onofrio RC, Winckler W, Weir BA, Beroukhim R, Pellman D, Levine DA, Lander ES, Meyerson M, Getz G: Absolute quantification of somatic DNA alterations in human cancer. Nat Biotechnol 2012, 30:413-421.

41. Oesper L, Mahmoody A, Raphael BJ: THetA: inferring intra-tumor heterogeneity from high-throughput DNA sequencing data. Genome Biol 2013, 14:R80.

42. Escohar M, West M: Bayesian Density Estimation and Inference Using Mixtures. J AM Statist Assoc 1995, 90:12

43. Roth A, Khattra J, Yap D, Wan A, Laks E, Biele J, Ha G, Aparicio S, Bouchard-Cote A, Shah SP: PyClone: statistical inference of clonal population structure in cancer. Nat Methods 2014, 11:396-398.

44. Schwarz G: Estimating the dimension of a model. Ann Stat 1978, 6:4.

45. Gould SJ, Eldredge N: Punctuated equilibrium comes of age. Nature 1993, 366:223-227.

46. Cibulskis K, Lawrence MS, Carter SL, Sivachenko A, Jaffe D, Sougnez C, Gabriel S, Meyerson M, Lander ES, Getz G: Sensitive detection of somatic point mutations in impure and heterogeneous cancer samples. Nat Biotechnol 2013, 31:213-219.

47. Team RC: R: A language and environment for statistical computing; 2012.

48. Olshen $A B$, Venkatraman $E S$, Lucito R, Wigler M: Circular binary segmentation for the analysis of array-based DNA copy number data. Biostatistics 2004, 5:557-572.

49. Parker JS, Mullins $M$, Cheang MC, Leung S, Voduc D, Vickery T, Davies S, Fauron C, He X, Hu Z, Quackenbush JF, Stijleman IJ, Palazzo J, Marron JS, Nobel AB, Mardis E, Nielsen TO, Ellis MJ, Perou CM, Bernard PS: Supervised risk predictor of breast cancer based on intrinsic subtypes. J Clin Oncol 2009, 27:1160-1167.

50. Andor N, Harness JV, Muller S, Mewes HW, Petritsch C: EXPANDS: expanding ploidy and allele frequency on nested subpopulations. Bioinformatics 2014, 30:50-60.

51. Yau C, Mouradov D, Jorissen RN, Colella S, Mirza G, Steers G, Harris A, Ragoussis J, Sieber O, Holmes CC: A statistical approach for detecting genomic aberrations in heterogeneous tumor samples from single nucleotide polymorphism genotyping data. Genome Biol 2010, 11:R92. 
52. Ding L, Ley TJ, Larson DE, Miller CA, Koboldt DC, Welch JS, Ritchey JK, Young MA, Lamprecht T, McLellan MD, McMichael JF, Wallis JW, Lu C, Shen D, Harris CC, Dooling DJ, Fulton RS, Fulton LL, Chen K, Schmidt H, Kalicki-Veizer J, Magrini VJ, Cook L, McGrath SD, Vickery TL, Wendl MC, Heath S, Watson MA, Link DC, Tomasson MH, et al: Clonal evolution in relapsed acute myeloid leukaemia revealed by whole-genome sequencing. Nature 2012, 481:506-510.

53. Hajirasouliha I, Mahmoody A, Raphael BJ: A combinatorial approach for analyzing intra-tumor heterogeneity from high-throughput sequencing data. Bioinformatics 2014, 30:i78-i86.

54. Jiao W, Vembu S, Deshwar AG, Stein L, Morris Q: Inferring clonal evolution of tumors from single nucleotide somatic mutations. BMC Bioinformatics 2014, 15:35.

55. Strino F, Parisi F, Micsinai M, Kluger Y: TrAp: a tree approach for fingerprinting subclonal tumor composition. Nucleic Acids Res 2013, 41:e165.

56. Xu Y, Muller P, Yuan Y, Gulukota K, Ji Y: MAD Bayes for tumor heterogeneity - feature allocatioon with exponential family sampling. arXiv:14025090 [statME] 2014.

57. Stephens PJ, Greenman CD, Fu B, Yang F, Bignell GR, Mudie LJ, Pleasance $E D$, Lau KW, Beare D, Stebbings LA, McLaren S, Lin ML, McBride DJ, Varela I, Nik-Zainal S, Leroy C, Jia M, Menzies A, Butler AP, Teague JW, Quail MA, Burton J, Swerdlow H, Carter NP, Morsberger LA, lacobuzio-Donahue C, Follows GA, Green AR, Flanagan AM, Stratton MR, et al: Massive genomic rearrangement acquired in a single catastrophic event during cancer development. Cell 2011, 144:27-40

58. Rausch T, Jones DT, Zapatka M, Stutz AM, Zichner T, Weischenfeldt J, Jager N, Remke M, Shih D, Northcott PA, Pfaff E, Tica J, Wang Q, Massimi L, Witt H, Bender S, Pleier S, Cin H, Hawkins C, Beck C, von Deimling A, Hans V, Brors B, Eils R, Scheurlen W, Blake J, Benes V, Kulozik AE, Witt O, Martin D, et al: Genome sequencing of pediatric medulloblastoma links catastrophic DNA rearrangements with TP53 mutations. Cell 2012, 148:59-71.

59. Molenaar JJ, Koster J, Zwijnenburg DA, van Sluis $P$, Valentijn $L J$, van der Ploeg I, Hamdi M, van Nes J, Westerman BA, van Arkel J, Ebus ME, Haneveld F, Lakeman A, Schild L, Molenaar P, Stroeken P, van Noesel MM, Ora I, Santo EE, Caron HN, Westerhout EM, Versteeg R: Sequencing of neuroblastoma identifies chromothripsis and defects in neuritogenesis genes. Nature 2012, 483:589-593.

60. Beerenwinkel N, Antal T, Dingli D, Traulsen A, Kinzler KW, Velculescu VE, Vogelstein B, Nowak MA: Genetic progression and the waiting time to cancer. PLoS Comput Biol 2007, 3:e225.

61. Bozic I, Antal T, Ohtsuki H, Carter H, Kim D, Chen S, Karchin R, Kinzler KW, Vogelstein B, Nowak MA: Accumulation of driver and passenger mutations during tumor progression. Proc Natl Acad Sci U S A 2010, 107:18545-18550.

62. Eldredge N, Gould SJ: Punctuated equilibria: an alternative to phyletic gradualism. In Models in Paleobiology. Edited by Schopf TJM. San Francisco, CA: Freeman Cooper; 1972

63. Cancer Genome Atlas Data Portal [https://tcga-data.nci.nih.gov/tcga/ dataAccessMatrix.htm]

doi:10.1186/s13059-014-0473-4

Cite this article as: $\mathrm{Li}$ and $\mathrm{Li}$ : A general framework for analyzing tumor subclonality using SNP array and DNA sequencing data. Genome Biology 2014 15:473.

\section{Submit your next manuscript to BioMed Central and take full advantage of:}

- Convenient online submission

- Thorough peer review

- No space constraints or color figure charges

- Immediate publication on acceptance

- Inclusion in PubMed, CAS, Scopus and Google Scholar

- Research which is freely available for redistribution 